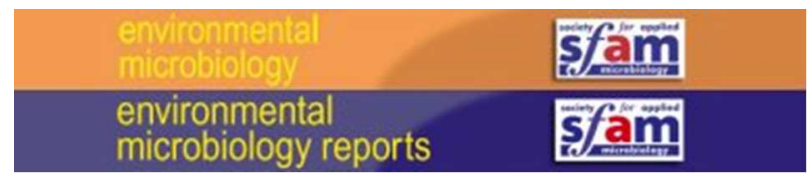

\title{
Fecal Clostridiales distribution and short-chain fatty acids reflect bowel habits in irritable bowel syndrome
}

\begin{tabular}{|r|l|}
\hline Journal: & Environmental Microbiology and Environmental Microbiology Reports \\
\hline Manuscript ID & EMI-2018-0551 \\
\hline Journal: & Environmental Microbiology \\
\hline Manuscript Type: & EMI - Research article \\
\hline Date Submitted by the Author: & 04-Apr-2018 \\
\hline Complete List of Authors: & $\begin{array}{l}\text { Gargari, Giorgio; University of Milan } \\
\text { Taverniti, Valentina; University of Milan } \\
\text { Guglielmetti, Simone; Universita degli Studi di Milano, Food Environmental } \\
\text { and Nutritional Sciences (DeFENS) }\end{array}$ \\
\hline Keywords: & $\begin{array}{l}\text { fecal microbiota, IBS, short chain fatty acids, Clostridiales, 16S rRNA gene } \\
\text { profiling }\end{array}$ \\
\hline &
\end{tabular}

SCHOLARONEm

Manuscripts 


\section{Fecal Clostridiales distribution and short-chain fatty acids reflect bowel habits in irritable bowel syndrome}

Giorgio Gargari ${ }^{1}$, Valentina Taverniti ${ }^{1}$, Claudio Gardana ${ }^{1}$, Cesare Cremon $^{2}$, Filippo Canducci ${ }^{3}$, Isabella Pagano $^{2}$, Maria Raffaella Barbaro ${ }^{2}$, Lara Bellacosa ${ }^{2}$, Anna Maria Castellazzi ${ }^{4}$, Chiara

Valsecchi ${ }^{5}$, Sara Carlotta Tagliacarne ${ }^{4}$, Massimo Bellini ${ }^{6}$, Lorenzo Bertani $^{6}$, Dario Gambaccini ${ }^{6}$,

Santino Marchi ${ }^{6}$, Michele Cicala ${ }^{7}$, Bastianello Germanà $^{8}$, Elisabetta Dal Pont ${ }^{8}$, Maurizio Vecchi ${ }^{9}$

Cristina Ogliari ${ }^{9}$, Walter Fiore ${ }^{10}$, Vincenzo Stanghellini ${ }^{2}$, Giovanni Barbara ${ }^{2}$, Simone

Guglielmetti $^{1 *}$

${ }^{1}$ Division of Food Microbiology and Bioprocesses, Department of Food, Environmental and Nutritional Sciences, University of Milan, Milan, Italy.

${ }^{2}$ Department of Medical and Surgical Sciences, Centre for Applied Biomedical Research, University of Bologna, Bologna, Italy.

${ }^{3}$ Dipartimento di biotecnologie e scienze della vita, Università degli Studi dell'Insubria, Varese, Italy.

${ }^{4}$ Department of Clinical Surgical Diagnostic and Pediatric Sciences, University of Pavia, Pavia, Italy.

${ }^{5}$ Department of Pediatrics, Fondazione IRCCS Policlinico San Matteo, Pavia, Italy

${ }^{6}$ Gastroenterology Unit, Department of Gastroenterology, University of Pisa, Pisa, Italy, Italy.

${ }^{7}$ Gastroenterology Unit, University Campus Bio-Medico of Rome, Rome, Italy.

${ }^{8}$ Gastroenterology Unit, S. Martino Hospital, Belluno, Italy.

${ }^{9}$ Gastroenterology and Digestive Endoscopy Unit, IRCCS Policlinico San Donato, San Donato Milanese, Italy.

${ }^{10}$ Sofar S.p.A., Trezzano Rosa, Italy.

* Address correspondence to Simone Guglielmetti, simone.guglielmetti@unimi.it.

Running Head: Fecal microbial ecosystem of IBS subtypes. 


\section{Originality-Significance Statement}

Irritable bowel syndrome (IBS) is a common, long-term condition that affects the large intestine and can occur with dramatically different symptoms from one person to another, especially in terms of their bowel habits. This study suggests, for the first time, that a network of correlations among (i) fecal Clostridiales bacteria, (ii) short-chain fatty acids, (iii) immunological factors, and (iv) clinical data may differentiate IBS subtypes. In this study, we propose that the bacterial taxa and SCFAs that distinguish the IBS categories may also serve as potential subtype-specific therapeutic targets for the management of IBS, which is the most prevalent functional gastrointestinal disorder in the Western world. 
5

\section{Summary}

Irritable bowel syndrome (IBS), a common functional gastrointestinal disorder, is classified according to bowel habits as IBS with constipation (IBS-C), with diarrhea (IBS-D), with alternating constipation and diarrhea (IBS-M), and unsubtyped (IBS-U). The mechanisms leading to the different IBS forms are mostly unknown. This study aims to evaluate whether specific fecal bacterial taxa and/or short-chain fatty acids (SCFAs) can be used to distinguish IBS subtypes and are relevant for explaining the clinical differences between IBS sub-categories. We characterized five fecal samples collected at 4-weeks intervals from 40 IBS patients by $16 \mathrm{~S}$ rRNA gene profiling and SCFA quantification. Finally, we investigated the potential correlations in IBS subtypes between the fecal microbial signatures and host physiological and clinical parameters. We found significant differences in the distribution of Clostridiales OTUs among IBS subtypes and reduced levels of SCFAs in IBS-C compared to IBS-U and IBS-D patients. Correlation analyses showed that the diverse representation of Clostridiales OTUs between IBS subtypes was associated with altered levels of SCFAs; furthermore, the same OTUs and SCFAs were associated with the fecal cytokine levels and stool consistency. Our results suggest that intestinal Clostridiales and SCFAs might serve as potential mechanistic biomarkers of IBS subtypes and represent therapeutic targets.

Key words: fecal microbiota, IBS, short-chain fatty acids, Clostridiales, 16S rRNA gene profiling. 


\section{Introduction}

Irritable bowel syndrome (IBS) is the most prevalent functional gastrointestinal disorder in the Western world. Although it does not have a lethal prognosis, IBS may significantly decrease the quality of life of patients depending on the severity of symptoms, which characteristically include abdominal pain, bloating, distension and altered bowel habits (Mearin et al., 2016).

IBS is a widely heterogeneous condition in terms of etiology, pathogenesis and clinical presentation. In a recent paper, Collins S. M. proposed to explain the intestinal dysfunctions associated with IBS through a gut-microbiota-centered model (Collins, 2014). According to this model, triggers such as extensive antibiotic use, infections and/or stress affect host functions, including mucin production, gut motility and hormone secretion, lead to dysbiosis (i.e., structural and functional alterations of the intestinal microbial ecosystem; IME), which in turn, promotes chronic gut dysfunction. Hence, Collins' model highlights the central role of the intestinal microbiota in IBS, in agreement with clinical evidence of the benefits generated by gut microbiotatargeting strategies, such as the use of the poorly absorbed antibiotic rifaximin (Li et al., 2016) and probiotics (O'Mahony et al., 2005; Guglielmetti et al., 2011). Accordingly, gut dysbiosis was often observed to be a common alteration associated with IBS (Taverniti and Guglielmetti, 2014; Zhuang et al., 2017). Contextually, several possible bacterial signatures have been proposed to distinguish IBS patients from healthy controls, such as increases in certain Ruminococcus phylotypes, reduction of bifidobacteria, or expansion of Proteobacteria and Veillonella spp. (Taverniti and Guglielmetti, 2014; Rajilic-Stojanovic et al., 2015). In addition, the available scientific literature also describes the significant role played by altered levels of short-chain fatty acids (SCFAs) in IBS (Ringel-Kulka et al., 2015; Camilleri et al., 2016; Farup et al., 2016). For instance, Farup and colleagues proposed that propionate and butyrate may act as discriminatory factors to differentiate healthy subjects from subjects with IBS (Ringel-Kulka et al., 2015). By contrast, in the study of Ringel-Kulka et al., SCFAs were found to discriminate IBS from healthy controls only when based on the subtype 
(Ringel-Kulka et al., 2015). Therefore, although they are recognized as biomarkers for IBS (Kim et al., 2017), SCFAs require further study to elucidate their actual role in IBS.

IBS is conventionally classified into four subtypes according to bowel habits: IBS with constipation (IBS-C), IBS with diarrhea (IBS-D), and IBS with alternating constipation and diarrhea (mixed IBS, IBS-M) as well as unsubtyped IBS (IBS-U) (Mearin et al., 2016). The diverse mechanisms underlying the pathophysiology of IBS subtypes remain unknown, and validated mechanistic biomarkers for the IBS subtypes are not available (Kim et al., 2017). IBS-subtype specific alterations of the intestinal microbiota have been reported (Malinen et al., 2005; Kassinen et al., 2007; Lyra et al., 2009; Carroll et al., 2010; Pozuelo et al., 2015; Tap et al., 2017). For instance, Tap et al. reported that IBS-D patients had more Methanobacteriales than patients with other IBS subtypes (Tap et al., 2017), whereas Pozuelo et al. reported evidence for an association between lower microbial diversity and a decreased abundance of butyrate-producing bacteria in patients with IBS-D and IBS-M (Pozuelo et al., 2015). However, data concerning the differences in the gut microbiota composition of the IBS subtypes are contradictory and are often based on methods that have low discriminatory power (Taverniti and Guglielmetti, 2014).

Inspired by the above considerations, this study was conducted to evaluate whether specific fecal bacterial taxa and/or colonic SCFAs can be used to distinguish IBS subtypes and are relevant for defining the mechanisms that lead to the clinical differences between IBS sub-categories. To fulfil this aim, we characterized the IME in IBS subtypes by means of $16 \mathrm{~S}$ ribosomal RNA (rRNA) gene profiling and SCFA quantification of fecal samples derived from a multicenter intervention trial that we recently performed to assess the effect of a probiotic preparation (L. casei $\mathrm{DG}^{\circledR}$; Lactobacillus paracasei CNCM I-1572) on the IBS symptoms of 40 patients (Cremon et al., 2017). In addition, the clinical and immunological data collected during the trial were used to investigate potential correlations in IBS subtypes between the IME and host physiological and clinical parameters, including bowel habits, depression/anxiety scores, and fecal levels of IgA and cytokines. We 
propose that the bacterial taxa and SCFAs that were identified can be used as to distinguish IBS subtypes and can also serve as potential therapeutic targets.

\section{Results}

The overall bacterial diversity of the fecal microbiota does not discriminate among IBS subtypes 16S rRNA gene profiling was performed on 198 fecal samples (5 fecal samples collected from 39 subjects and 3 fecal samples from a subject who dropped out after visit V3), generating a total of $16,963,222$ filtered high-quality sequence reads (a mean of 138,413 reads per sample). Rarefaction curves demonstrated that most fecal microbiota diversity had been covered (not shown). The Unifrac algorithm was used to investigate inter-sample $\beta$-diversity. The intra-patient variability observed among the five samples analyzed is shown in Supplementary figure S1 according to the two main components extracted.

In the subsequent analyses, besides considering the data of a single $16 \mathrm{~S}$ rRNA gene profiling determination per subject at baseline (single profiling data, corresponding to the data obtained from the analysis of the first fecal sample per subject, collected at visit V1; $n=40$ ), we also performed the analyses with data corresponding to the medians of five $16 \mathrm{~S}$ rRNA gene profiling determinations per patient (median profiling data, corresponding to the median value of data obtained from the analysis of all fecal samples per subject, which were collected at visits from V1 to V5; $\mathrm{n}=39$ ).

Afterwards, we investigated the $\beta$-diversity of the different types of IBS. This analysis revealed that both weighted and unweighted Unifrac cannot distinguish fecal samples on the basis of IBS subtypes either with individual (Supplementary Figures S2) or median (Supplementary Figures S1 and Fig. 1) profiling data.

Next, intra-subject taxonomic richness and evenness ( $\alpha$-diversity) were analyzed using five algorithms; namely, observed OTUs, Chao1, Faith's Phylogenetic Diversity, and Shannon and 
130 Simpson indexes. The $\alpha$-diversity indexes of the IBS subtypes did not significantly differ with 131 either individual or median profiling data (Supplementary Figure S3).

132 The microbiota profiling data were then stratified by enterotyping based on the relative 133 abundances of the bacterial genera (Gargari et al., 2016). An optimal number of three groups of 134 samples was generated; nonetheless the Silhouette coefficient, which validates the consistency 135 within groups of data, was too low to consider the clustering reliable (Supplementary Figure S4). 136 Notably, the taxonomic overview of all 198 IBS fecal samples analyzed revealed that the first seven 137 most abundant genera belonged to the Firmicutes Gram-positive order Clostridiales (Supplementary 138 Figure S5A); in particular, Clostridiales accounted for approximately $75 \%$ of the detected bacteria; 139 in contrast, the relative abundance of members of the order Bacteroidales was lower than $10 \%$ 140 (Supplementary Figure S5A). On the contrary, in our previous studies, we found that Bacteroidales 141 (particularly the genera Bacteroides and Prevotella) were the dominant genera of the fecal 142 microbiota in healthy volunteers (Ferrario et al., 2014; Gargari et al., 2016). Therefore, at the end of 143 the IBS trial, we analyzed additional fecal samples collected from 16 healthy adults through 16S 144 rRNA gene profiling and adopting the same protocol used for the IBS samples with the sole aim of assessing whether the observed expansion of Clostridiales compared to Bacteroidales is a bona fide microbiological feature of the investigated IBS patients. The results showed that Clostridiales are largely dominant also in the feces of control subjects (Fig. S5B), demonstrating that the alteration of the Clostridiales/Bacteroidales ratio observed in IBS samples depended on technical issues, most 149 likely the protocol used for the extraction of metagenomic DNA from the feces. Indeed, differently 150 from the present study, in our previous works, we extracted fecal metagenomic DNA using a 151 commercial kit that did not include a cell-breaking step using bead beater, plausibly resulting in the underestimation of the Gram-positive bacteria (e.g., Clostridiales), which have a stronger cell walls than Gram-negative cells (e.g., Bacteroidales). 
Overall, these data indicate that the bacterial ecological diversity indexes of the fecal microbiota do not vary significantly among IBS subtypes. The results of this study showed a general dominance of Clostridiales in the fecal samples collected from both IBS and control subjects.

\section{IBS-C and IBS-D fecal samples are differently enriched in OTUs ascribed to Clostridiales}

Subsequently, microbiomic data were examined with the DESeq2 negative binomial distribution method to infer differential relative abundances at the OTU level between IBS subtypes (IBS-C, $\mathrm{n}=12$; IBS-D, $\mathrm{n}=11$; IBS-M, $\mathrm{n}=3$; IBS-U, $\mathrm{n}=11$ ). The analysis was performed both on $\mathrm{V} 1$ and V1-5 profiling data; the IBS-M subtype was excluded because too few patients $(n=3)$ had this subtype to allow the identification of significant differences. We found that several OTUs discriminated among the three IBS subtypes considered (Fig. 2 and Supplementary Figure 6). A summary of the number of significantly different OTUs was plotted as a Venn diagram (Fig. 2A). Specifically, the analysis of median profiling data revealed 26 significantly different OTUs between IBS-U and IBSC, 11 of which were also found while analyzing individual profiling data (Fig. 2B and Supplementary Figure 6); 19 OTUs distinguished IBS-U from IBS-D, 6 of which were also found while analyzing individual profiling data (Fig. 2B and Supplementary Figure 6). The greatest number of dissimilarities was found between IBS-C and IBS-D: 85 OTUs had significantly different relative abundances, 39 of which were also found while analyzing individual profiling data (Fig. 2B and Supplementary Figure 6). Most of the discriminating OTUs were taxonomically ascribed to the order Clostridiales (Fig. 2B and Supplementary Figure 6); in particular, IBS-C was distinguished from IBS-D by numerous OTUs associated with Clostridiales belonging to the families Ruminococcaceae (in particular, the genus Ruminococcus) and Lachnospiraceae. In addition, two OTUs ascribed to Bifidobacterium adolescentis were increased in IBS-C, whereas OTUs associated with the order Bacteroidales (i.e., Bacteroides caccae, Parabacteroides distasonis and Prevotella copri) and to the Firmicutes species Eubacterium biforme were enriched in the IBSD samples (Fig. 2B and Supplementary Figure 6). 
Overall, these results indicate that the fecal microbiota of IBS-C and IBS-D are characterized by

a different distribution of Clostridiales taxonomic units, whereas the fecal microbiota of the IBS-U samples possessed compositional features that were intermediate between those of the IBS-C and IBS-D samples.

\section{IBS subtypes are characterized by altered fecal levels of short-chain fatty acids}

The intestinal levels of the short-chain fatty acids (SCFAs) acetate, butyrate, isobutyrate, valerate, isovalerate and propionate were quantified in the IBS fecal samples and used to characterize the IBS subtypes. To determine the fecal microbiota composition, the SCFAs were analyzed considering the levels determined in a single fecal sample per patient (single analysis SCFA levels, $\mathrm{n}=37$; Supplementary Figure 7) and the median values of five measurements per patient (median SCFA levels, n=37; Fig. 3A). SCFAs were also quantified in the IBS-M fecal samples, but this subgroup was excluded from the statistical analyses due to the limited number of patients $(n=3)$. In addition, the SCFA levels in the IBS samples were compared with those of healthy subjects $(n=25)$, which were determined in a previous study (Gargari et al., 2016).

We found that the fecal levels of SCFAs clearly distinguished the IBS-C samples from the IBSD and IBS-U samples. In detail, the levels of acetate, butyrate, propionate and valerate were significantly higher in IBS-D than in IBS-C. In addition, fecal concentrations of acetate, butyrate and propionate were higher in IBS-U than in IBS-C. Compared to all IBS samples considered together, the fecal level of acetate was significantly lower in IBS-C, whereas the fecal level of valerate was significantly higher in IBS-D (Fig. 3A). No significant differences among the IBS subgroups were observed for isobutyrate and isovalerate (Fig. 3A).

We did not find significant differences between the IBS samples and healthy controls with the sole exception of isovalerate, which was lower in IBS. Nonetheless, notably, numerous significant differences emerged when the IBS subtypes were considered separately. We found that acetate and propionate where significantly higher in IBS-D compared to healthy controls, whereas acetate and 
valerate were significantly lower in IBS-C than controls; globally, the total concentration of SCFAs was significantly higher in IBS-D and lower in IBS-C compared to healthy controls, whereas IBS-U levels were not dissimilar from the controls (Fig. 3A and Supplementary Figure 7).

Subsequently, a principal component analysis (PCA) was performed to discriminate samples based on fecal SCFA levels. As evidenced by the PCA bi-plot depicted in Fig. 3B, increased levels of acetate, butyrate and propionate characterized the IBS-D samples and distinguished them from the IBS-C samples $(\mathrm{R}=0.133 ; \mathrm{P}=0.011$ according to ANOSIM test); on the other hand, IBS-U and the healthy controls are located in an intermediate area of the plot.

Overall, these data indicate that significant differences in the fecal levels of SCFAs can be found between healthy adults and IBS patients only if IBS subtypes are considered; specifically, IBS-D samples are characterized by the increase of and IBS-C samples are characterized by the decrease of the fecal levels of SCFAs. Contrarily, fecal SCFAs were not dissimilar between the IBS-U and control samples.

\section{The intestinal microbial ecosystem reflects clinical features of IBS subtypes}

Finally, we performed correlation analyses between the fecal microbial ecology data and clinical parameters of the IBS patients to find relationships between IME and the clinical parameters. The correlation analysis was performed as described in the materials and methods section using a nonparametric correlation test (Spearman and Kendall). To this end, we used as predictors the fecal levels of SCFAs or the relative abundances of the OTUs that we found to be significantly different between IBS subtypes; the dependent variables considered were SCFAs, Bristol stool scale data (to assess bowel habits), abdominal pain/discomfort score, fecal levels of IgA and cytokines (TGF $\beta$, IL6, IL8, IL10, IL12, IFN $\gamma$, and TNF $\alpha$ ), and HADS and SF-12 questionnaire data (to evaluate anxiety and depression, and quality of life, respectively) (Cremon et al., 2017). As with the previous analyses, correlations were estimated based on data collected at a single time point (V1) and on median data for multiple time points (V1-V5). 
We found that host parameters were significantly correlated with numerous OTUs (Fig. 2B). Notably, we found that most Clostridiales OTUs that were enriched in IBS-C samples were negatively correlated with the fecal SCFAs propionate and butyrate, whereas several Clostridiales OTUs that were overrepresented in IBS-D were positively correlated with acetate and valerate (Fig. 2B). Moreover, most IBS-C-enriched OTUs that were inversely linked to SCFAs were positively correlated with several cytokines (particularly IL10) and were negatively correlated with IgA. Conversely, several IBS-D-enriched OTUs that were positively associated with SCFAs were also positively correlated with the fecal type as determined using the Bristol stool scale (Fig. 2B). Accordingly, we found a positive correlation between the fecal type and acetate, butyrate and valerate (Fig. 4). In addition, notably, IgA resulted positively correlated with evacuation frequency and negatively correlated with IL10 and TNF $\alpha$ (Fig. 4).

Overall, these results indicate that the differential representation of Clostridiales OTUs between IBS subtypes is associated with altered levels of intestinal SCFAs; then, in turn, both OTUs and SCFAs are associated with stool consistency.

\section{Discussion}

The primary aim of the present study was to characterize the gut microbiota in IBS subtypes. To achieve this, we carried out 16S rRNA gene profiling and SCFAs quantification in 198 fecal samples obtained from 40 IBS patients enrolled in 5 Italian hospitals (Cremon et al., 2017).

Temporal instability is a distinguishing feature of the intestinal microbiota associated with IBS (Matto et al., 2005; Maukonen et al., 2006; Durban et al., 2013); for this reason, it was suggested that studies aimed at characterizing the gut microbiota in IBS should include multiple time points (Collins, 2014). Accordingly, in this study, we based microbiota analyses on data obtained from five fecal samples collected at 4-week intervals from each patient. These samples derived from a randomized cross-over intervention trial that assessed the clinical efficacy of a probiotic product. Although we are aware that the treatment may have affected the intestinal microbiota of IBS 
258

259

260

patients, we believe that the benefits of using five different fecal samples per subject are greater than the possible bias incurred and may permit a more reliable identification of gut microbiota biomarkers for IBS subtypes, for the following reason: All the analyses were carried out considering only data at baseline (i.e., originating from the analysis of the fecal samples collected at visit V1, when no product or placebo had yet been administered to the patients; single sample data analysis); single sample data analysis implies a mistake due to the great variability of the gut microbiota in IBS subjects, whereas the analysis with the median data of five samples per subject may determine an error due to the subject-dependent response to the probiotic treatment. The two potential errors are compensated by the combined use of the results derived from the analyses of single and median data. We believe, therefore, that those OTUs and SCFAs that yielded significantly different results between IBS subtypes based on the analysis of both data populations can be very plausibly considered valid microbial signatures.

Several studies focused on the characterization of the microbiota in IBS, with particular attention being paid to the identification of microbial markers distinguishing this dysfunction from the healthy condition (Zhuang et al., 2017); however, much less attention has been spent to compare the IMEs of IBS subtypes. In this context, Tap and collaborators recently reported that neither the richness nor the variability of the intestinal microbiota differed among IBS groups (Tap et al., 2017). Accordingly, we did not find significant differences in either $\alpha$ - or $\beta$-diversity among the IBS subtypes. In a previous study, Jeffery et al. (Jeffery et al., 2012) used pyrosequencing of the 16S rRNA gene to determine the microbiota composition in fecal specimens from 37 IBS patients. Notably, they identified distinct IBS patient subsets; however, these did not correspond to the traditional IBS subtypes (Jeffery et al., 2012). On the contrary, in the present study, we found that the relative abundance of numerous OTUs were significantly different among the IBS subtypes. In particular, we report here that major differences exist in Clostridiales OTUs between IBS-C and 
IBS-D feces; conversely, IBS-U fecal samples differed much less from IBS-C and IBS-D in terms of OTUs.

A rapidly expanding body of literature is demonstrating the clinical efficacy of dietary patterns based on drastically reducing fermentable oligo-, di-, mono-saccharides and polyols (the lowFODMAP diet) (Eswaran et al., 2016). Reportedly, FODMAPs are preferential fermentation substrates for the intestinal Clostridiales bacteria (Flint et al., 2012); accordingly, several trials have demonstrated that these bacteria may be affected by reduced FODMAP intake (Chumpitazi et al., 2014; Halmos et al., 2015; McIntosh et al., 2016). Therefore, we speculate that Clostridiales bacteria in the gut of IBS patients may represent a therapeutic target modulated by the lowFODMAP diet.

Many OTUs that distinguished IBS-C from IBS-D samples belonged to the Clostridiales families Ruminococcaceae and Lachnospiraceae. The importance of these gut bacteria in IBS was also evidenced by the study of Tap et al., who defined a composite gut microbial signature for IBS severity constituted by 90 OTUs; at the family level, these principally included OTUs within Lachnospiraceae and Ruminococcaceae (Tap et al., 2017). Lachnospiraceae and Ruminococcaceae, which are the most commonly retrieved families in the active intestinal microbiota (Peris-Bondia et al., 2011), are considered the principal intestinal microorganisms that degrade plant carbohydrates (Wolin et al., 2003; Chassard et al., 2007; Flint et al., 2012), producing SCFAs as their main catabolites (Flint et al., 2012). These bacterial families include the most important butyrateproducing microorganisms in the human gut such as the genera Faecalibacterium and Roseburia (Barcenilla et al., 2000; Louis et al., 2010) as well as bacteria that can produce acetate from reductive acetogenesis (Bernalier et al., 1996; Rey et al., 2010) and butyrate or propionate from lactate utilization (Duncan et al., 2004; Rios-Covian et al., 2016).

Considering the above-mentioned literature, the observed differential OTU distribution between IBS-C and IBS-D samples suggest that the IBS subtypes have dissimilar fecal levels of SCFAs. 
Accordingly, we found significantly lower levels of acetate, butyrate, propionate and valerate in IBS-C samples. Notably, these results were also confirmed when considering the data calculated as the medians of five determinations per subject over approximately 4 months, confirming the observed differences in SCFAs between IBS sub-categories.

The scientific literature on intestinal SCFAs in IBS is quite limited and contradictory, showing no altered, augmented, or decreased levels compared to healthy controls (Mortensen et al., 1987; Treem et al., 1996; Tana et al., 2010; Halmos et al., 2014; Rajilic-Stojanovic et al., 2015). In our study, we did not find significant differences in the fecal levels of the main SCFAs when the data from all IBS samples were compared with the fecal SCFA concentrations found in healthy adults as determined using the same protocol in a recent study (Gargari et al., 2016). Nonetheless, substantial differences emerged when the IBS subtypes were considered independently. Our data are in accordance with the study of Ringel-Kulka et al. (Ringel-Kulka et al., 2015), in which IBS-D patients $(n=42)$ were shown to have significantly higher fecal levels of acetate, propionate and butyrate than IBS-C patients $(n=26)$. Interestingly, in this study, the authors also found that fecal SCFA concentrations were negatively correlated with colon transit time. This result is potentially in agreement with the positive correlation we found between fecal type (determined using the Bristol stool scale) and acetate, butyrate and valerate levels.

The link between colon transit time and intestinal SCFAs in IBS subtypes can be explained by two possible opposite mechanisms (Ringel-Kulka et al., 2015): (1) compared to IBS-C, IBS-D patients are characterized by increased colonic fermentation, which leads to higher fecal levels of SCFAs, thereby stimulating intestinal motility (Fukumoto et al., 2003) and reducing transit time; or (2) decreased transit time in IBS-D patients slows down SCFA absorption, leading to higher SCFA concentrations in the feces compared to those in IBS-C patients. Here, we showed that several OTUs were significantly enhanced in IBS-D compared to IBS-C, and this was correlated positively with fecal levels of SCFAs (especially acetate) and fecal type; at the same time, a number of OTUs that were expanded in IBS-C were inversely correlated with SCFAs. Nevertheless, both explanatory 
scenarios are still valid. On one hand, it is possible that the different distribution of intestinal

334 bacteria is responsible for the dissimilar concentration of SCFAs in IBS subtypes. On the other hand, it can be speculated that bacteria in the colon may be differently affected by modified intestinal transit (for instance, due to variable adhesion abilities and/or cell reproduction rates

337 among the diverse bacteria) with a consequent modification of the relative distribution of bacterial taxa in feces. However, two facts might support the first scenario: (i) most of the fecal bacteria that distinguish IBS-C from IBS-D are members of taxa known to be SCFA producers and (ii) it is known that SCFAs stimulate colonic motility and may increase the osmotic load leading to diarrhea (Fritz et al., 2005). In summary, we think it is reasonable to hypothesize a self-perpetuating mechanism in which an initial modified colon transit time (determined by any possible trigger, such as gut infections or intensive antibiotic use) gives rise to intestinal dysbiosis, which, in turn, leads to altered intestinal levels of SCFAs that may exacerbate or maintain the altered intestinal motility.

Reportedly, immune system activation is involved in the pathophysiology of IBS (Barbara et al., 2011). In particular, cytokines are mediators of immune responses that can be involved in motor dysfunctions and visceral pain (Dinan et al., 2006). In this study, correlation analyses revealed significant positive associations of IgA and IFN $\gamma$ with evacuation frequency. Little information is available in the scientific literature concerning intestinal IgA in IBS; nonetheless, our results are consistent with those of Wahnschaffe et al., who reported a significant decrease in stool frequency and intestinal IgA levels under a gluten-free diet in a subgroup of celiac IBS patients (Wahnschaffe et al., 2001). In addition, IFN $\gamma$ was shown to be increased in the gut of IBS patients and to reduce the expression of the serotonin transporter (SERT), thereby resulting in increased serotonin levels and motility (Barbaro et al., 2016).

\section{Conclusions}

This study suggests that the altered distribution of bacteria inside the Gram-positive order Clostridiales can be used to distinguish the intestinal microbial ecosystem of IBS subtypes and 
359

360

361

362

363

364

365

366

367

368

369

370

371

372

373

374

375

376

377

378

379

380

381

382

383

384

plausibly contributes to the observed altered fecal levels of SCFAs. The main limitation of this study is the limited sample size. Nonetheless, we believe that the repeated measures per patient combined with the bioinformatics analysis that we used was suitable to identify key microbial signatures that can define the IBS types. Although we are aware that the results presented here are not proof of a cause-effect relationship between IME and clinical outputs in IBS, we hypothesize that intestinal Clostridiales and colonic SCFAs can be used as mechanistic biomarkers of IBS subtypes and also potentially represent therapeutic targets. In addition, this study supports the notion that distinct therapeutic approaches should be developed for the different IBS subtypes.

\section{Experimental procedures}

\section{Patients and study protocol}

Eligible patients with symptoms meeting the Rome III criteria for IBS diagnosis were recruited in five Italian hospitals as previously described (Cremon et al., 2017). In brief, the inclusion criteria comprised a positive diagnosis of IBS (of any subtype), age between 18 and 65 years, negative colonoscopy or barium enema examination within the previous 2 years, and negative relevant additional screening or consultation whenever appropriate. Patients were excluded if they were pregnant, breast-feeding, or not using reliable methods of contraception. The exclusion criteria also included the presence of intestinal organic diseases, such as celiac disease, as ascertained by the detection of anti-transglutaminase antibodies; diverticular disease; or inflammatory bowel diseases (IBDs; e.g., Crohn's disease, ulcerative colitis, infectious colitis, ischemic colitis, or microscopic colitis); previous major abdominal surgery; untreated food intolerance, such as ascertained or suspected lactose intolerance as defined by an anamnestic evaluation or, if appropriate, a lactose breath test; consumption of probiotics or topical and/or systemic antibiotic therapy during the month before study enrolment; systematic/frequent consumption (i.e., once weekly or more frequent) of contact laxatives; presence of any relevant organic, systemic, or metabolic disease as assessed by the medical history, appropriate consultations, and laboratory tests; or abnormal 
laboratory values deemed to be clinically significant on the basis of predefined values. Upon enrollment, all patients were asked to maintain their habitual diet. The gender, age and subtypes of the enrolled population are reported in Supplementary Table 1. The enrolled patients were included in a multicenter, randomized, double-blind, cross-over, placebo-controlled, pilot trial (PROBE-IBS trial, registered under the ClinicalTrial.gov No. NCT02371499), whose primary endpoint was the assessment of the effect of Lactobacillus paracasei CNCM I-1572 (LCDG) on the IBS clinical symptoms. The design and results of the PROBE-IBS trial are described in (Cremon et al., 2017). In brief, PROBE-IBS consisted of a two-week run-in phase, after which the volunteers were randomly assigned to take either LCDG twice daily for four weeks or a placebo (treatment A). At the end of this phase, the patients entered a four-week washout period before crossing over to the alternate treatment (twice daily for four weeks: treatment B), followed by a four-week follow-up period. The patients collected and delivered a fecal sample before and after treatment A (at visits V1 and V2, respectively), before and after treatment B (at visits V3 and V4, respectively), and after the followup period (visit V5). The number of patients was calculated before the recruitment started.

\section{Collected data and missing samples}

A total of 40 IBS patients (IBS-C, $n=12$; IBS-D, $n=14$; IBS-M, n=3; IBS-U, $n=11$ ) were included in the study. IBS subtypes were classified according to the Rome III criteria and based on Bristol Stool Form scale characteristics (Longstreth et al., 2006). Information and biological specimens were collected every four weeks at five consecutive time points (visits V1-V5) according to the trial design described by Cremon et al. (Cremon et al., 2017). One participant (belonging to the IBS-D subgroup) dropped out after visit V3 and, consequently, 198 fecal samples were collected. 16S rRNA gene profiling analyses were performed on all samples, whereas SCFAs were quantified in the 5 fecal samples of 37 patients (i.e., a total of 185 samples; IBS-C, n=12; IBS-D, n=11; IBS-M, $\mathrm{n}=3$; IBS-U, $\mathrm{n}=11$ ) due to insufficient specimens. Data from Bristol stool scale, anxiety/depression scales, and $\operatorname{IgA}$ and cytokine data were available as described in (Cremon et al., 2017). Correlation 
analyses were performed using data from a subgroup of 150 samples (30 patients) instead of 200 (40 patients) because we removed samples with immunological data below the detection limit.

After the end of the study, we also included 16 control subjects (i.e., non-diseased adults without IBS). Controls were recruited to match the parameters of age and sex of the IBS patients (Supplementary Table 1).

\section{Profiling of the fecal microbiota composition}

Fecal samples were collected by patients and kept in refrigerator until delivery to the laboratory. Once delivered, stools were stored at $-80^{\circ} \mathrm{C}$ until the beginning of the analysis. Metagenomic DNA was extracted from about $200 \mathrm{mg}$ of feces using the PowerSoil ${ }^{\circledR}$ DNA Isolation Kit (MO BIO Laboratories) according to the manufacturer's instructions. Subsequently, the bacterial community structure was profiled by 16S rRNA gene profiling. In brief, Probio_Uni and Probio_Rev primers were used to amplify a partial region of the $16 \mathrm{~S}$ rRNA encompassing the V3 variable region (Gargari et al., 2016). Next, amplicons were sequenced using Illumina MiSeq System and the resulting sequence reads were managed by means of the bioinformatic pipeline Quantitative Insights Into Microbial Ecology (QIIME) version 1.7.0 (Caporaso et al., 2010) with the GreenGenes database (version 13.5), which allowed clustering of sequences into operational taxonomic units (OTUs). Metadata have been deposited in the European Nucleotide Archive (ENA) of the European Bioinformatics Institute under accession code PRJEB18753.

\section{Quantification of fecal short-chain fatty acids (SCFAs)}

SCFAs were quantified in the fecal samples as previously described (Gargari et al., 2016). In brief, $100 \mathrm{mg}$ of stools were suspended in $2 \mathrm{ml}$ of $0.001 \%$ formic acid, vortexed for $1 \mathrm{~min}$, and centrifuged at $1000 \mathrm{xg}$ for $2 \mathrm{~min}$ at $4{ }^{\circ} \mathrm{C}$. Supernatant was recovered and pellet was extracted again as described above. Then, the two supernatants were combined and the volume adjusted to $5 \mathrm{ml}$ with $0.001 \%$ formic acid solution. All extracts were stored at $-20{ }^{\circ} \mathrm{C}$ until analysis, which was performed by UPLC-HR-MS on Acquity UPLC separation module (Waters, Milford, MA, USA) coupled with an Exactive Orbitrap MS through an HESI-II probe for electrospray ionization 
436 (Thermo Scientific, San Jose, CA, USA). Column, ion source and interface conditions were 437 reported in (Gargari et al., 2016). Elution was carried out at a flow-rate of $0.2 \mathrm{ml} / \mathrm{min}$ with solvents $0.001 \% \mathrm{HCOOH}$ in MilliQ-treated water (solvent A) and $\mathrm{CH}_{3} \mathrm{OH}: \mathrm{CH}_{3} \mathrm{CN}(1: 1 \mathrm{v} / \mathrm{v}$, solvent B), using the following elution gradient: $0 \% \mathrm{~B}$ for $4 \mathrm{~min}, 0-15 \% \mathrm{~B}$ in $6 \mathrm{~min}, 15-20 \% \mathrm{~B}$ in $5 \mathrm{~min}, 20 \%$ for $13 \mathrm{~min}$, and then return to initial conditions in $1 \mathrm{~min}$. Subsequently, the UPLC eluate was analyzed in full scan MS in the range $50-130 \mathrm{~m} / \mathrm{z}$ as described elsewhere (Gargari et al., 2016). External calibration curves were prepared with reagents from Sigma-Aldrich (Milan, Italy) to quantify acetic, butyric, isobutyric, isovaleric, propionic, and valeric acids in fecal samples. SCFA concentrations were expressed in mmol per kilogram of wet feces.

\section{Statistical analysis}

Data concerning the intestinal microbial ecosystem (16S rRNA gene profiles and SCFA quantifications) were analyzed using R statistics software (version 3.1.2) and QIIME. Significant differences were determined using the Wilcoxon-Mann-Whitney test for unpaired data. Significant differences at the OTU level between IBS subtypes were determined using differential gene expression analysis based on the negative binomial distribution method (R/Bioconductor DESeq2 package); an FDR-adjusted $p$-value ( $q$-value) with a cut off value of 0.1 was used for the threshold (Love et al., 2014). DESeq2 analysis was performed on both single (V1) and median (V1-5) microbiomic data. For the analysis of the median profiling data, the DESq2 model was applied to the medians of the reads counts at five time points per subject. Correlation analyses were performed using the Kendall and Spearman formulas with the items specified in the text as predictors and dependent variables. Significance was set at $\mathrm{P} \leq 0.05$; significance in the range $0.05<\mathrm{P}<0.10$ was accepted as a trend. UNIFRAC algorithms were used to study the inter-sample diversity of the fecal microbiota composition. To define enterotypes, microbiota profiling data were analyzed based on genus relative abundance using the JSD distance and the Partitioning Around Medoids (PAM) algorithm (Gargari et al., 2016). Significant differences between groups of samples in principal 

similarities).

\section{Conflict of Interest}

No conflict of interest is known to the authors.

\section{Acknowledgments and funding}

This study was financially supported by Sofar S.p.A.

\section{References}

Barbara, G., Cremon, C., Carini, G., Bellacosa, L., Zecchi, L., De Giorgio, R. et al. (2011) The immune system in irritable bowel syndrome. J Neurogastroenterol Motil 17: 349-359.

Barbaro, M.R., Di Sabatino, A., Cremon, C., Giuffrida, P., Fiorentino, M., Altimari, A. et al. (2016) Interferongamma is increased in the gut of patients with irritable bowel syndrome and modulates serotonin metabolism. Am J Physiol Gastrointest Liver Physiol 310: G439-447.

Barcenilla, A., Pryde, S.E., Martin, J.C., Duncan, S.H., Stewart, C.S., Henderson, C., and Flint, H.J. (2000) Phylogenetic relationships of butyrate-producing bacteria from the human gut. Appl Environ Microbiol 66: 1654-1661.

Bernalier, A., Willems, A., Leclerc, M., Rochet, V., and Collins, M.D. (1996) Ruminococcus hydrogenotrophicus sp. nov., a new $\mathrm{H} 2 / \mathrm{CO} 2$-utilizing acetogenic bacterium isolated from human feces. Arch Microbiol 166: 176-183.

Camilleri, M., Oduyebo, I., and Halawi, H. (2016) Chemical and molecular factors in irritable bowel syndrome: current knowledge, challenges, and unanswered questions. Am J Physiol Gastrointest Liver Physiol 311: G777-G784.

Caporaso, J.G., Kuczynski, J., Stombaugh, J., Bittinger, K., Bushman, F.D., Costello, E.K. et al. (2010) QIIME allows analysis of high-throughput community sequencing data. Nat Methods 7: 335-336.

Carroll, I.M., Chang, Y.H., Park, J., Sartor, R.B., and Ringel, Y. (2010) Luminal and mucosal-associated intestinal microbiota in patients with diarrhea-predominant irritable bowel syndrome. Gut Pathog 2: 19.

Chassard, C., Goumy, V., Leclerc, M., Del'homme, C., and Bernalier-Donadille, A. (2007) Characterization of the xylan-degrading microbial community from human faeces. FEMS Microbiol Ecol 61: 121-131.

Chumpitazi, B.P., Hollister, E.B., Oezguen, N., Tsai, C.M., McMeans, A.R., Luna, R.A. et al. (2014) Gut microbiota influences low fermentable substrate diet efficacy in children with irritable bowel syndrome. Gut Microbes 5: 165-175.

Collins, S.M. (2014) A role for the gut microbiota in IBS. Nat Rev Gastroenterol Hepatol 11: 497-505.

Cremon, C., Guglielmetti, S., Gargari, G., Taverniti, V., Castellazzi, A.M., Valsecchi, C. et al. (2017) Effect of Lactobacillus paracasei CNCM I-1572 on symptoms, gut microbiota, short chain fatty acids, and immune activation in patients with irritable bowel syndrome: A pilot randomized clinical trial. United European Gastroenterology Journal: 10.1177/2050640617736478.

Dinan, T.G., Quigley, E.M., Ahmed, S.M., Scully, P., O'Brien, S., O'Mahony, L. et al. (2006) Hypothalamicpituitary-gut axis dysregulation in irritable bowel syndrome: plasma cytokines as a potential biomarker? Gastroenterology 130: 304-311.

Duncan, S.H., Louis, P., and Flint, H.J. (2004) Lactate-utilizing bacteria, isolated from human feces, that produce butyrate as a major fermentation product. Appl Environ Microbiol 70: 5810-5817. 
502

503

504

505

506

507

508

509

510

511

512

513

514

515

516

517

518

519

520

521

522

523

524

525

526

527

528

529

530

531

532

533

534

535

536

537

538

539

540

541

542

543

544

545

546

547

548

549

550

551

552
Durban, A., Abellan, J.J., Jimenez-Hernandez, N., Artacho, A., Garrigues, V., Ortiz, V. et al. (2013) Instability of the faecal microbiota in diarrhoea-predominant irritable bowel syndrome. FEMS Microbiol Ecol 86: 581-589.

Eswaran, S.L., Chey, W.D., Han-Markey, T., Ball, S., and Jackson, K. (2016) A Randomized Controlled Trial Comparing the Low FODMAP Diet vs. Modified NICE Guidelines in US Adults with IBS-D. Am J Gastroenterol 111: 1824-1832.

Farup, P.G., Rudi, K., and Hestad, K. (2016) Faecal short-chain fatty acids - a diagnostic biomarker for irritable bowel syndrome? BMC Gastroenterol 16: 51.

Ferrario, C., Taverniti, V., Milani, C., Fiore, W., Laureati, M., De Noni, I. et al. (2014) Modulation of fecal Clostridiales bacteria and butyrate by probiotic intervention with Lactobacillus paracasei DG varies among healthy adults. J Nutr 144: 1787-1796.

Flint, H.J., Scott, K.P., Duncan, S.H., Louis, P., and Forano, E. (2012) Microbial degradation of complex carbohydrates in the gut. Gut Microbes 3: 289-306.

Fritz, E., Hammer, H.F., Lipp, R.W., Hogenauer, C., Stauber, R., and Hammer, J. (2005) Effects of lactulose and polyethylene glycol on colonic transit. Aliment Pharmacol Ther 21: 259-268.

Fukumoto, S., Tatewaki, M., Yamada, T., Fujimiya, M., Mantyh, C., Voss, M. et al. (2003) Short-chain fatty acids stimulate colonic transit via intraluminal 5-HT release in rats. Am J Physiol Regul Integr Comp Physiol 284: R1269-1276.

Gargari, G., Taverniti, V., Balzaretti, S., Ferrario, C., Gardana, C., Simonetti, P., and Guglielmetti, S. (2016) Consumption of a Bifidobacterium bifidum Strain for 4 Weeks Modulates Dominant Intestinal Bacterial Taxa and Fecal Butyrate in Healthy Adults. Appl Environ Microbiol 82: 5850-5859.

Guglielmetti, S., Mora, D., Gschwender, M., and Popp, K. (2011) Randomised clinical trial: Bifidobacterium bifidum MIMBb75 significantly alleviates irritable bowel syndrome and improves quality of life--a doubleblind, placebo-controlled study. Aliment Pharmacol Ther 33: 1123-1132.

Halmos, E.P., Power, V.A., Shepherd, S.J., Gibson, P.R., and Muir, J.G. (2014) A diet low in FODMAPs reduces symptoms of irritable bowel syndrome. Gastroenterology 146: 67-75 e65.

Halmos, E.P., Christophersen, C.T., Bird, A.R., Shepherd, S.J., Gibson, P.R., and Muir, J.G. (2015) Diets that differ in their FODMAP content alter the colonic luminal microenvironment. Gut 64: 93-100.

Jeffery, I.B., O'Toole, P.W., Ohman, L., Claesson, M.J., Deane, J., Quigley, E.M., and Simren, M. (2012) An irritable bowel syndrome subtype defined by species-specific alterations in faecal microbiota. Gut 61: 9971006.

Kassinen, A., Krogius-Kurikka, L., Makivuokko, H., Rinttila, T., Paulin, L., Corander, J. et al. (2007) The fecal microbiota of irritable bowel syndrome patients differs significantly from that of healthy subjects. Gastroenterology 133: 24-33.

Kim, J.H., Lin, E., and Pimentel, M. (2017) Biomarkers of Irritable Bowel Syndrome. J Neurogastroenterol Motil 23: 20-26.

Li, J., Zhu, W., Liu, W., Wu, Y., and Wu, B. (2016) Rifaximin for Irritable Bowel Syndrome: A Meta-Analysis of Randomized Placebo-Controlled Trials. Medicine (Baltimore) 95: e2534.

Longstreth, G.F., Thompson, W.G., Chey, W.D., Houghton, L.A., Mearin, F., and Spiller, R.C. (2006) Functional bowel disorders. Gastroenterology 130: 1480-1491.

Louis, P., Young, P., Holtrop, G., and Flint, H.J. (2010) Diversity of human colonic butyrate-producing bacteria revealed by analysis of the butyryl-CoA:acetate CoA-transferase gene. Environ Microbiol 12: 304314.

Love, M.I., Huber, W., and Anders, S. (2014) Moderated estimation of fold change and dispersion for RNAseq data with DESeq2. Genome Biol 15: 550.

Lyra, A., Rinttila, T., Nikkila, J., Krogius-Kurikka, L., Kajander, K., Malinen, E. et al. (2009) Diarrhoeapredominant irritable bowel syndrome distinguishable by $16 \mathrm{~S}$ rRNA gene phylotype quantification. World J Gastroenterol 15: 5936-5945.

Malinen, E., Rinttila, T., Kajander, K., Matto, J., Kassinen, A., Krogius, L. et al. (2005) Analysis of the fecal microbiota of irritable bowel syndrome patients and healthy controls with real-time PCR. Am J Gastroenterol 100: 373-382. 
Matto, J., Maunuksela, L., Kajander, K., Palva, A., Korpela, R., Kassinen, A., and Saarela, M. (2005) Composition and temporal stability of gastrointestinal microbiota in irritable bowel syndrome--a longitudinal study in IBS and control subjects. FEMS Immunol Med Microbiol 43: 213-222.

Maukonen, J., Satokari, R., Matto, J., Soderlund, H., Mattila-Sandholm, T., and Saarela, M. (2006) Prevalence and temporal stability of selected clostridial groups in irritable bowel syndrome in relation to predominant faecal bacteria. J Med Microbiol 55: 625-633.

McIntosh, K., Reed, D.E., Schneider, T., Dang, F., Keshteli, A.H., De Palma, G. et al. (2016) FODMAPs alter symptoms and the metabolome of patients with IBS: a randomised controlled trial. Gut.

Mearin, F., Lacy, B.E., Chang, L., Chey, W.D., Lembo, A.J., Simren, M., and Spiller, R. (2016) Bowel Disorders. Gastroenterology.

Mortensen, P.B., Andersen, J.R., Arffmann, S., and Krag, E. (1987) Short-chain fatty acids and the irritable bowel syndrome: the effect of wheat bran. Scand J Gastroenterol 22: 185-192.

O'Mahony, L., McCarthy, J., Kelly, P., Hurley, G., Luo, F., Chen, K. et al. (2005) Lactobacillus and bifidobacterium in irritable bowel syndrome: symptom responses and relationship to cytokine profiles. Gastroenterology 128: 541-551.

Peris-Bondia, F., Latorre, A., Artacho, A., Moya, A., and D'Auria, G. (2011) The active human gut microbiota differs from the total microbiota. PLoS One 6: e22448.

Pozuelo, M., Panda, S., Santiago, A., Mendez, S., Accarino, A., Santos, J. et al. (2015) Reduction of butyrateand methane-producing microorganisms in patients with Irritable Bowel Syndrome. Sci Rep 5: 12693.

Rajilic-Stojanovic, M., Jonkers, D.M., Salonen, A., Hanevik, K., Raes, J., Jalanka, J. et al. (2015) Intestinal microbiota and diet in IBS: causes, consequences, or epiphenomena? Am J Gastroenterol 110: 278-287.

Rey, F.E., Faith, J.J., Bain, J., Muehlbauer, M.J., Stevens, R.D., Newgard, C.B., and Gordon, J.I. (2010) Dissecting the in vivo metabolic potential of two human gut acetogens. J Biol Chem 285: 22082-22090.

Ringel-Kulka, T., Choi, C.H., Temas, D., Kim, A., Maier, D.M., Scott, K. et al. (2015) Altered Colonic Bacterial Fermentation as a Potential Pathophysiological Factor in Irritable Bowel Syndrome. Am J Gastroenterol 110: $1339-1346$.

Rios-Covian, D., Ruas-Madiedo, P., Margolles, A., Gueimonde, M., de Los Reyes-Gavilan, C.G., and Salazar, N. (2016) Intestinal Short Chain Fatty Acids and their Link with Diet and Human Health. Front Microbiol 7: 185.

Tana, C., Umesaki, Y., Imaoka, A., Handa, T., Kanazawa, M., and Fukudo, S. (2010) Altered profiles of intestinal microbiota and organic acids may be the origin of symptoms in irritable bowel syndrome. Neurogastroenterol Motil 22: 512-519, e114-515.

Tap, J., Derrien, M., Tornblom, H., Brazeilles, R., Cools-Portier, S., Dore, J. et al. (2017) Identification of an Intestinal Microbiota Signature Associated With Severity of Irritable Bowel Syndrome. Gastroenterology 152: $111-123$ e118.

Taverniti, V., and Guglielmetti, S. (2014) Methodological issues in the study of intestinal microbiota in irritable bowel syndrome. World J Gastroenterol 20: 8821-8836.

Treem, W.R., Ahsan, N., Kastoff, G., and Hyams, J.S. (1996) Fecal short-chain fatty acids in patients with diarrhea-predominant irritable bowel syndrome: in vitro studies of carbohydrate fermentation. $J$ Pediatr Gastroenterol Nutr 23: 280-286.

Wahnschaffe, U., Ullrich, R., Riecken, E.O., and Schulzke, J.D. (2001) Celiac disease-like abnormalities in a subgroup of patients with irritable bowel syndrome. Gastroenterology 121: 1329-1338.

Wolin, M.J., Miller, T.L., Collins, M.D., and Lawson, P.A. (2003) Formate-dependent growth and homoacetogenic fermentation by a bacterium from human feces: description of Bryantella formatexigens gen. nov., sp. nov. Appl Environ Microbiol 69: 6321-6326.

Zhuang, X., Xiong, L., Li, L., Li, M., and Chen, M. (2017) Alterations of gut microbiota in patients with irritable bowel syndrome: A systematic review and meta-analysis. J Gastroenterol Hepatol 32: 28-38. 
601

602

603

604

605

606

607

608

609

610

611

612

613

614

615

616

617

618

619

620

621

622

623

624

\section{Legends}

Fig. 1. Ecological $\beta$-diversity of the fecal microbiota in the IBS subtypes. Principal coordinates analysis of weighted (A) and unweighted (B) Unifrac distances based on the medians of OTU abundances related to five fecal samples per IBS patient $(n=39)$. The first two coordinates (PC1 and PC2) are displayed with the percentage of variance explained in brackets.

Fig. 2. OTUs distinguishing IBS subtypes determined using the DESeq2 negative binomial distribution method on the 16S rRNA gene profiling data of five fecal samples per patient. A, Venn diagrams summarizing the number of OTUs that discriminate IBS subtypes on the basis of $16 \mathrm{~S}$ rRNA gene profiling data of a single sample (single profiling data) and five samples (median profiling data) per patient. C, IBS with constipation (IBS-C); D, IBS with diarrhea (IBS-D); U, unsubtyped IBS (IBS-U). Overrepresented OTUs are reported with the same letter color indicating the IBS subtype. B, IBS subtype-discriminating OTUs according to median profiling data and their correlation with host physiological and clinical parameters. OTUs that also distinguished IBS subtypes according to the single profiling data analysis are reported in bold. The heatmap on the left represents the mean normalized relative abundances of the reported OTUs. The taxonomic lineage of each taxon is shown; p, phylum; c, class; o, order; f, family; g, genus; s, species. Positive fold changes (shown on a red background) designate OTU overrepresentation in the IBS subtype indicated in the column to the left of the Normalized Base Mean; negative fold changes (shown on a green background) designate the OTU overrepresentation in the IBS subtype indicated in the column to the right of the Normalized Base Mean. The heatmap in the right panel represents the Rvalue of Spearman's correlation between the OTU and host parameters. Asterisks indicate the Kendall rank correlation: *, $\mathrm{P}<0.05 ; * *, \mathrm{P}<0.01$; ***, $\mathrm{P}<0.001$. Black margins around boxes indicate that the correlations remained significant (according to Kendall's $p$ value) when determined using individual sample data. 
625 Fig. 3. Short-chain fatty acids (SCFAs) in fecal samples of IBS patients. A, Medians of SCFA 626 concentrations in five fecal samples (wet weight) per IBS patient ( $\mathrm{n}=37$; IBS-D, $\mathrm{n}=11$; IBS-C, $627 \mathrm{n}=12$; IBS-U, $\mathrm{n}=11$; IBS-M, $\mathrm{n}=3$ ) and in healthy controls ( $\mathrm{n}=25$; data from (Gargari et al., 2016)).

628 Significance was determined using the Mann-Whitney test; *, $\mathrm{P}<0.05$; **, $\mathrm{P}<0.01$. B, Principal 629 component analysis (PCA) biplot of SCFAs (represented by arrows) and IBS patients. The first two 630 coordinates (PC1 and PC2) are displayed with the percentage of variance explained in brackets.

631 Fig. 4. Correlations among short-chain fatty acids (SCFAs), physiological data and clinical 632 parameters. The analysis was performed using median data. The heatmap represents the R-value of 633 Spearman's correlation. Asterisks indicate the Kendall rank correlation: *, $\mathrm{P}<0.05 ; * *, \mathrm{P}<0.01 ; * * *$, $634 \mathrm{P}<0.001$. Black margins around boxes indicate that the correlations remained significant (according 635 to Kendall's $\mathrm{p}$ value) when determined using individual sample data. 
A Weighted UNIFRAC

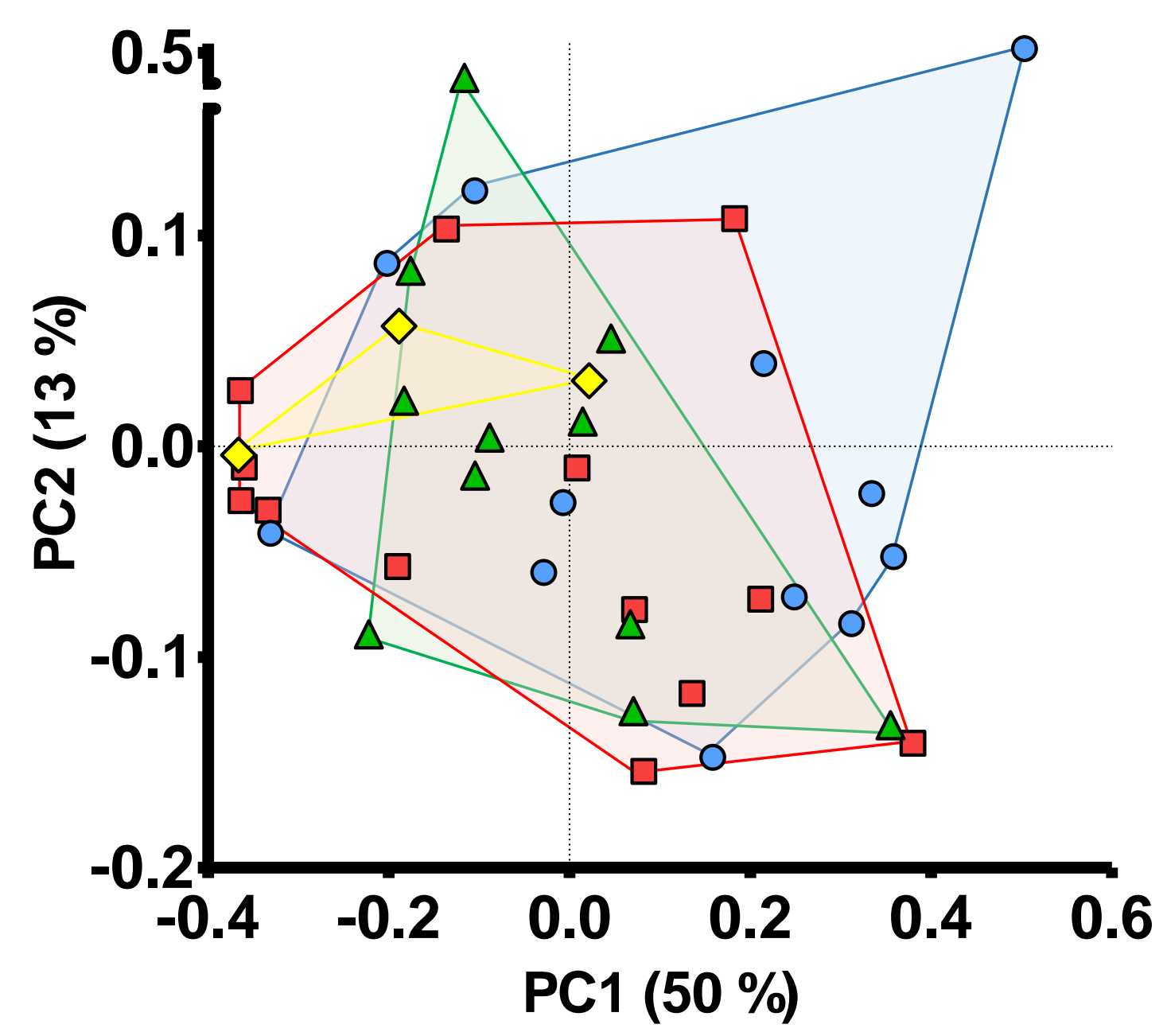

\section{B Unweighted UNIFRAC}

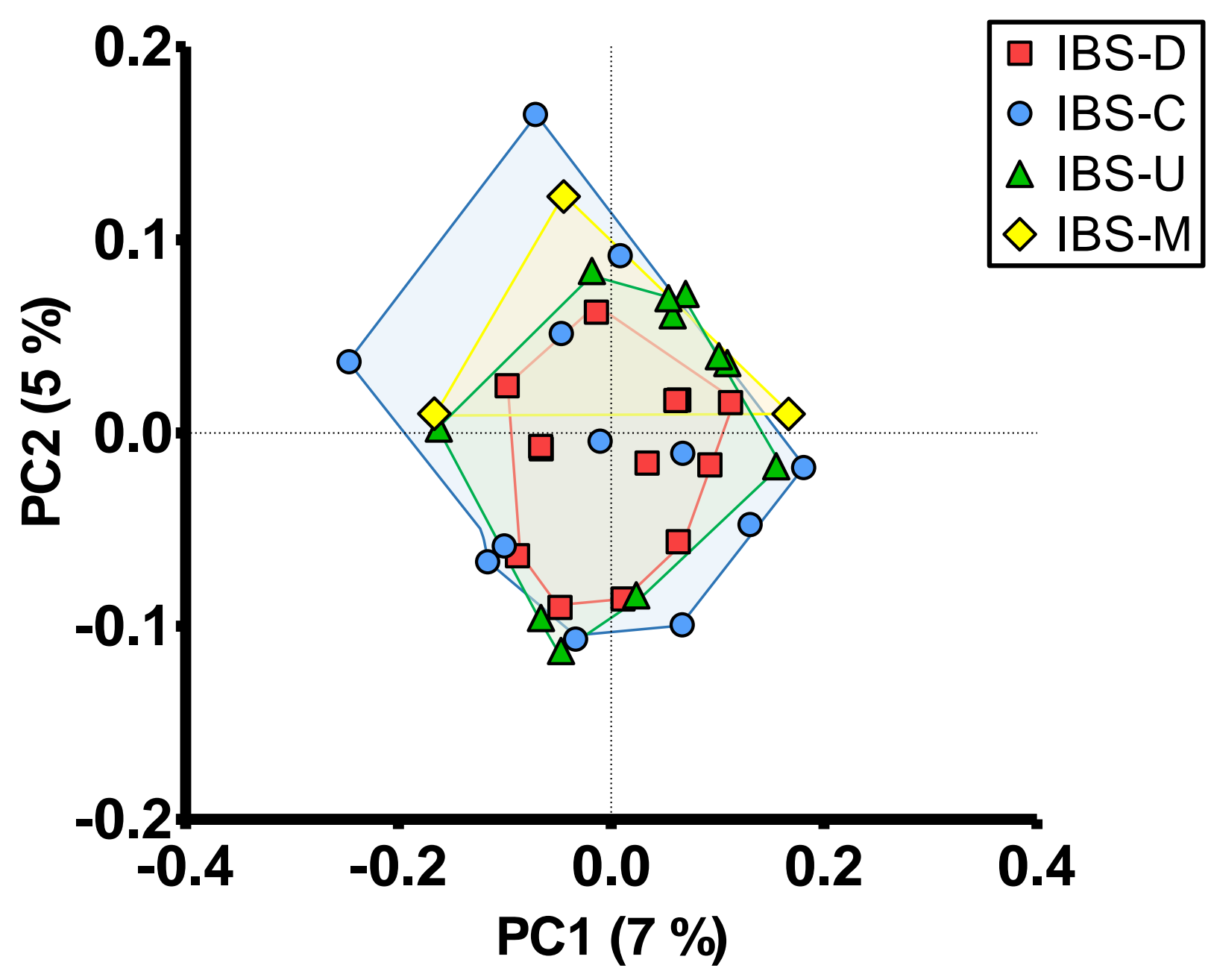


Fig. 2A

\author{
Single \\ profiling data \\ Median \\ profiling data
}

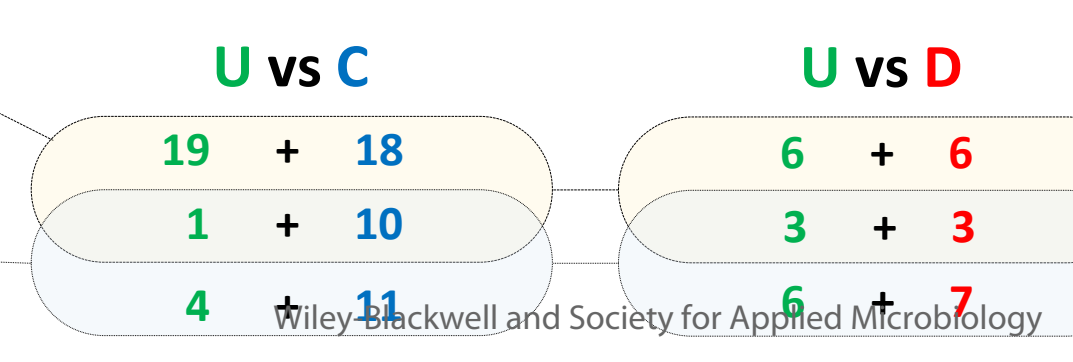

C vs D

$32+22$

$30+9$

$17+29$ 


\begin{tabular}{|c|c|c|}
\hline \multirow[b]{2}{*}{ оTU } & $\begin{array}{l}\text { Normalized } \\
\text { Base Mean }\end{array}$ & \multirow[b]{2}{*}{$\begin{array}{l}\log 2 \text { Fold } \\
\text { Change }\end{array}$} \\
\hline & IBS-U IBS-C & \\
\hline OTU2423305 & & -2.62 \\
\hline ncur_OTU43120 & & 1.90 \\
\hline OTU4336943 & & -3.02 \\
\hline OTU552988 & & -2.62 \\
\hline отU537219 & & -2.30 \\
\hline отU700540 & & -2.20 \\
\hline отU815179 & & -2.11 \\
\hline nr_OTU249 & & -1.74 \\
\hline отU310178 & & -3.72 \\
\hline OTU345944 & & -2.22 \\
\hline отU410242 & & -1.86 \\
\hline OTU555547 & & -1.64 \\
\hline ОTU192240 & & -2.13 \\
\hline OTU548021 & & -2.69 \\
\hline OTU436032 & & -2.00 \\
\hline ОтU349257 & & -2.30 \\
\hline отU369827 & & -3.99 \\
\hline отU342947 & & -2.13 \\
\hline OTU564320 & & -1.99 \\
\hline отU369602 & & -4.38 \\
\hline OTU197943 & & -2.65 \\
\hline OTU441934 & & 2.49 \\
\hline отU174516 & & 2.00 \\
\hline OTU196332 & & 1.60 \\
\hline OTU369763 & & 1.99 \\
\hline OTU1820513 & & -2.08 \\
\hline
\end{tabular}

\begin{tabular}{|c|c|c|}
\hline \multirow[b]{2}{*}{ отU } & $\begin{array}{l}\text { Normalized } \\
\text { Base Mean }\end{array}$ & \multirow[b]{2}{*}{$\begin{array}{c}\text { log2 Fold } \\
\text { Change }\end{array}$} \\
\hline & IBS-U IBS-D & \\
\hline$\overline{\text { OTU530653 }}$ & & -3.00 \\
\hline OTU107044 & & -2.05 \\
\hline OTU4035247 & & -1.90 \\
\hline OTU185061 & & -3.22 \\
\hline OTU192226 & & -2.10 \\
\hline OTU341777 & & -2.20 \\
\hline OTU524884 & & -3.41 \\
\hline nr_OTU436 & & -2.13 \\
\hline OTU197105 & & -1.87 \\
\hline OTU191421 & & 2.09 \\
\hline OTU360890 & & 2.32 \\
\hline OTU583974 & & 2.00 \\
\hline $\begin{array}{l}\text { OTU584978 }\end{array}$ & & 2.44 \\
\hline OTU183532 & & 2.13 \\
\hline OTU287608 & & 2.77 \\
\hline OTU342427 & & 2.28 \\
\hline OTU592616 & & 2.23 \\
\hline отU2з3953 & & 2.03 \\
\hline OTU1820513 & & -2.37 \\
\hline
\end{tabular}

padj Tanonomy

1.1E-02 p_Actinobacteria;c_Coriobacteriia;o_Coriobacteriales;__Coriobacteriaceae;g_;

2.0E-02 P_Actinobacteria;__Actinobacteria;__Actinomycetales;__Actinomycetaceae;__Actinomyces;s_

$1.5 \mathrm{E}-03$ P_Bacteroidetes;c_Bacteroidia;0_Bacteroidales; $;$ _Rikenellaceae;__;

$6.0 \mathrm{E}-04$ p_Firmicutes;c_Clostridia;o_Clostridiales;f_;g_;

9.4E-03 p_Firmicutes;c_Clostridia;o_Clostridiales; ;_;_; $;$

$1.4 \mathrm{E}-02$ p_Firmicutes;c_Clostridia;o_Clostridiales; $\mathrm{f}_{-} ; \mathrm{g}_{-} ; \mathrm{s}_{-}$

$1.1 \mathrm{E}-02$ P_Firmicutes;c_Clostridia;o_Clostridiales;f_; $;$ _;s

$1.4 \mathrm{E}-02$ P_Firmicutes;c_Clostridia;o_Clostridiales;__;g_; _

$6.9 \mathrm{E}-07$ P_Firmicutes;c_Clostridia;o_Clostridiales;__Christensenellaceae;g_;

4.3E-02 P_Firmicutes;C_Clostridia;o_Clostridiales;__Christensenellaceae; __; _

3.1E-02 P_Firmicutes;c_Clostridia;o_Clostridiales;f_Christensenellaceae;g_;s

1.4E-02 P_Firmicutes;__Clostridia;o_Clostridiales;__Christensenellaceae;__;_

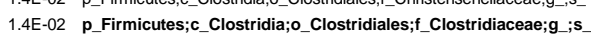

$1.5 \mathrm{E}-03$ P_Firmicutes;C_Clostridia;o_Clostridiales;__Lachnospiraceae;g_; _

$1.4 \mathrm{E}-02$ P_Firmicutes;C_Clostridia;__Clostridiales;__Lachnospiraceae;g_Blautia;s_

$1.4 \mathrm{E}-02$ P_Firmicutes;C_Clostridia;o_Clostridiales;__Lachnospiraceae;g_Lachnospira;s

2.6E-07 p_Firmicutes;c_Clostridia;o_Clostridiales;f_Ruminococcaceae; __;

2.4E-02 p_Firmicutes;c_Clostridia;o_Clostridiales;__Ruminococcaceae;g_;s

2.1E-02 p_Firmicutes;c_Clostridia;o_Clostridiales;__Ruminococcaceae; $g_{-}$;

$2.6 \mathrm{E}-07$ p_Firmicutes;c_Clostridia;o_Clostridiales;__Ruminococcaceae;g_Ruminococcus;s_

$1.1 \mathrm{E}-02$ P_Firmicutes;c_Clostridia;o_Clostridiales;__Ruminococcaceae;g_Ruminococcus;s_

2.5E-02 P_Firmicutes;C_Clostridia;o_Clostridiales;_;_g_; _

3.3E-02 p_Firmicutes;c_Clostridia;o_Clostridiales;f_Clostridiaceae;g_Clostridium;s

2.7E-02 P_Firmicutes;C_Clostridia;o_Clostridiales;__Lachnospiraceae;__Blautia;s

3.3E-02 p_Firmicutes;c_Erysipelotrichi;o_Erysipelotrichales;f_Erysipelotrichaceae; $g_{\text {CCOprobacillus;s }}$

$2.7 \mathrm{E}-02$ P_Proteobacteria;c_Betaproteobacteria;o_Burkholderiales;f_Alcaligenaceae;g_Sutterella;s

padj Tanonomy

4.4E-04 p Bacteroidetes;c Bacteroidia;o Bacteroidales;f Prevotellaceae;g_Prevotella;s_copri

$2.0 \mathrm{E}-02$ P_Bacteroidetes;c_Bacteroidia;o_Bacteroidales; $;$ _Rikenellaceae

4.5E-02 P_Bacteroidetes;c_Bacteroidia;0_Bacteroidales;__Rikenellaceae; __;__

$8.1 \mathrm{E}-05$ P_Firmicutes;__CClostridia;__Clostridiales;_;;_;_;_

$2.0 E-02$ P_Firmicutes;C_Clostridia;o_Clostridiales;f_Lachnospiraceae;g_Ruminococcus];s

2.6E-02 P_Firmicutes;c_Clostridia;o_Clostridiales;__Ruminococcaceae;g_Oscillospira;s_

8.7E-04 p_Firmicutes;c_Erysipelotrichi;o_Erysipelotrichales;f_Erysipelotrichaceae;g_[Eubacterium];s_biforme

8.1E-03 p Firmicutes;c_Erysipelotrichi;o_Erysipelotrichales;f Erysipelotrichaceae;g[Eubacterium];s biforme

3.2E-02 p Firmicutes;c_Erysipelotrichi;o Erysipelotrichales;_EErysipelotrichaceae;g_[Eubacterium];s_biforme

4.5E-02 P_Firmicutes;__CClostridia;o_Clostridiales;_;; __; _

3.0E-02 P_Firmicutes;_C_Clostridia;__CClostridiales;__;g_; _

5.7E-03 P_Firmicutes;C_Clostridia;o_Clostridiales;__Lachnospiraceae;___s_

$5.7 E-03$ P_Firmicutes;c_Clostridia;o_Clostridiales;__Ruminococcaceae;g_;s

$1.5 \mathrm{E}-02$ p_Firmicutes;c_Clostridia;o_Clostridiales;__Ruminococcaceae;g_Ruminococcus;s_

3.9E-03 p_Firmicutes;c_Clostridia;o_Clostridiales; $;$ Ruminococcaceae; $g$ Ruminococcus;s

$1.9 E-02$ p_Firmicutes;c_Clostridia;o_Clostridiales;__Veillonellaceae;g_Veillonella;s_dispar

$2.2 \mathrm{E}-02$ P_Firmicutes;C_Erysipelotrichi;o_Erysipelotrichales;_Erysipelotrichaceae;g_;__

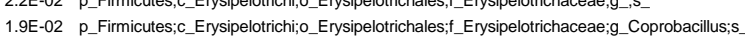

8.1E-03 P_Proteobacteria;c_Betaproteobacteria;o_Burkholderiales;__Alcaligenaceae;g_Sutterella;
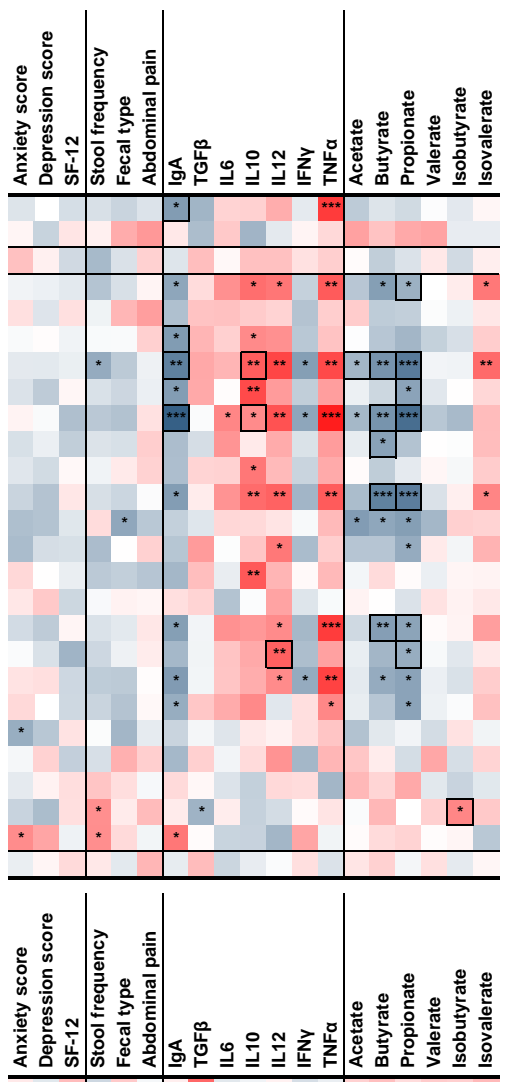

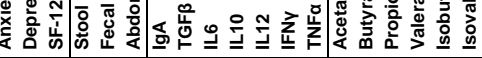

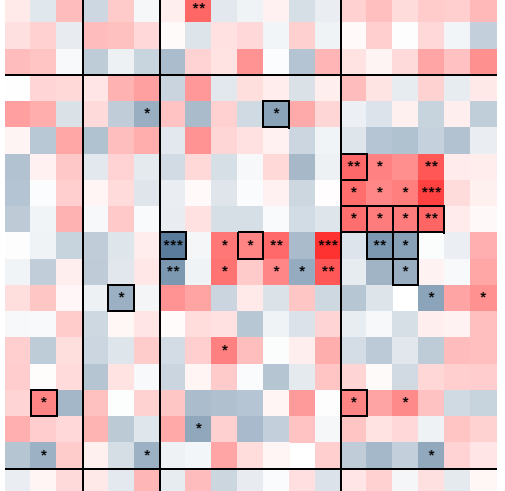


OTU364341

OTU582652

OTU356011

ncur_OTU21274

OTU183532

ncur_OTU51465

OTU287608

OTU369602

OTU342427

BS-C IBS-D log2 Fold

padj Tanonomy

\begin{tabular}{|c|c|c|}
\hline 1.63 & $3.3 \mathrm{E}-02$ & P_Actinobacteria;__Actinobacteria \\
\hline 1.80 & $2.0 \mathrm{E}-02$ & P_Actinobacteria;c_Actinobacteria;o_Bifidobacteriales; $;$ _Bifidobacteriaceae;g_Bifidobacterium;s_adolescentis \\
\hline 1.83 & $1.0 \mathrm{E}-02$ & P_Actinobacteria;c_Actinobacteria;o_Bifidobacteriales;f_Bifidobacteriaceae;g_Bifidobacterium;s_adolescentis \\
\hline-1.74 & $2.9 \mathrm{E}-02$ & p_Bacteroidetes;c_Bacteroidia;o_Bacteroidales;__Bacteroidaceae;__Bacteroides;s__ \\
\hline-1.58 & $4.5 \mathrm{E}-02$ & P_Bacteroidetes;c_Bacteroidia;o_Bacteroidales;f_Bacteroidaceae;g_Bacteroides;s_ \\
\hline-2.18 & 8.4E-03 & P_Bacteroidetes;c_Bacteroidia;o_Bacteroidales;__Bacteroidaceae;g_Bacteroides;s_caccae \\
\hline-2.02 & $1.8 \mathrm{E}-02$ & P_Bacteroidetes;c_Bacteroidia;o_Bacteroidales;f_Porphyromonadaceae;g_Parabacteroides;s_distasonis \\
\hline-3.03 & $6.2 \mathrm{E}-05$ & P_Bacteroidetes;c_Bacteroidia;o_Bacteroidales;__Prevotellaceae;g_Prevotella;s_copri \\
\hline-2.43 & $2.0 \mathrm{E}-03$ & p_Bacteroidetes;c_Bacteroidia;o_Bacteroidales;_Prevotellaceae;g_Prevotella;s_copri \\
\hline-2.38 & $2.1 \mathrm{E}-02$ & P_Bacteroidetes;c_Bacteroidia;o_Bacteroidales;_Prevotellaceae;g_Prevotella;s_copri \\
\hline 1.57 & $3.2 \mathrm{E}-02$ & P_Bacteroidetes;c_Bacteroidia;o_Bacteroidales;__Bacteroidaceae;__Bacteroides;s_ \\
\hline 2.12 & $6.0 \mathrm{E}-03$ & _Bacteroidia;0_Bacteroidales;;_[Barnesiellaceae];g_;s_ \\
\hline
\end{tabular}

$6.0 \mathrm{E}-03$ P_Bacteroidetes;C_Bacteroidia;o_Bacteroidales;_[Barnesiellaceae];g_;

3.5E-04 p_Firmicutes;C_Clostridia;o_Clostridiales;_;_;_;s

$2.5 \mathrm{E}-03$ p_Firmicutes;c_Clostridia;o_Clostridiales; $\mathbf{f}_{-} ; \mathrm{g}_{-} ; \mathbf{s}_{-}$

4.4E-02 P_Firmicutes;c_Clostridia;__Clostridiales;__Clostridiaceae;g_Clostridium;s

4.4E-02 P_Firmicutes;c_Clostridia;o_Clostridiales;_LLachnospiraceae

3.4E-02 P_Firmicutes;___Clostridia;o_Clostridiales;__Lachnospiraceae

2.2E-02 P_Firmicutes;c_Clostridia;o_Clostridiales;__Lachnospiraceae

2.1E-02 p_Firmicutes;c_Clostridia;o_Clostridiales;__Lachnospiraceae;g_s

$1.4 \mathrm{E}-02$ p_Firmicutes;c_Clostridia;o_Clostridiales;f_Lachnospiraceae;g_;s

2.1 E-03 P_Firmicutes;C_Clostridia;o_Clostridiales;f_Lachnospiraceae;g_[Ruminococcus];s

$1.6 \mathrm{E}-03$ P_Firmicutes;c_Clostridia;_CClostridiales;__Lachnospiraceae;g_Blautia

$1.2 \mathrm{E}-02$ P_Firmicutes;c_Clostridia;o_Clostridiales;f_Lachnospiraceae;g_Blautia;s

1.2E-02 P_Firmicutes;c_Clostridia;o_Clostridiales;f_Ruminococcaceae;g_; _ _

$1.5 \mathrm{E}-02$ P_Firmicutes;c_Clostridia;o_Clostridiales;__Ruminococcaceae;__; _

4.1E-02 p_Firmicutes;c_Clostridia;o_Clostridiales;__Ruminococcaceae; __;s

3.3E-02 P_Firmicutes;c_Clostridia;o_Clostridiales;__Ruminococcaceae;g_;s

$1.7 \mathrm{E}-02$ P_Firmicutes;c_Clostridia;o_Clostridiales;__Ruminococcaceae;g_;

2.3E-02 P_Firmicutes;c_Clostridia;o_Clostridiales;_Ruminococcaceae;g_;

4.5E-02 P_Firmicutes;__Clostridia;o_Clostridiales;__Ruminococcaceae;g_;

4.4E-02 p_Firmicutes;c_Clostridia;o_Clostridiales;__Ruminococcaceae;g_Faecalibacterium;s_prausnitzii

9.9E-03 p_Firmicutes;C_Clostridia;o_Clostridiales;__Ruminococcaceae;g_Faecalibacterium;s_prausnitzii

$3.8 \mathrm{E}-02$ P_Firmicutes;c_Clostridia;o_Clostridiales;__Ruminococcaceae;g_Faecalibacterium;s_prausnitzii

3.4E-02 P_Firmicutes;C_Clostridia;o_Clostridiales;__Ruminococcaceae;g_Faecalibacterium;s_prausnitzii

4.4E-02 p_Firmicutes;C_Clostridia;__Clostridiales;__Ruminococcaceae;g_Faecalibacterium;s_prausnitzii

$2.8 \mathrm{E}-02$ P_Firmicutes;C_Clostridia;o_Clostridiales;__Ruminococcaceae; ;_Ruminococcus;s_

2.2E-02 p_Firmicutes;c_Erysipelotrichi;__Erysipelotrichales;__Erysipelotrichaceae;g_Eubacterium];s_biforme

$1.3 \mathrm{E}-02$ P_Firmicutes;C_Erysipelotrichi;o_Erysipelotrichales;_fErysipelotrichaceae;g_[Eubacterium];__biforme

2.1E-02 p_Firmicutes;c_Erysipelotrichi;o_Erysipelotrichales; fEEysipelotrichaceae;g_Eubacterium];s_biforme

8.3E-03 p_Firmicutes;c_Erysipelotrichi;o_Erysipelotrichales; $f_{\text {EErysipelotrichaceae; _C_Coprobacillus;s }}$

9.1E-03 P_Firmicutes;c_Clostridia;o_Clostridiales

4.1E-02 P_Firmicutes;c_Clostridia;__Clostridiales

$2.0 \mathrm{E}-02$ P_Firmicutes;c_Clostridia;o_Clostridiales;f_;g_;s

4.1E-02 P_Firmicutes;C_Clostridia;o_Clostridiales;f; ; _; __

6.0E-03 p_Firmicutes;c_Clostridia;o_Clostridiales;_;;_;s

7.4E-04 P_Firmicutes;c_Clostridia;o_Clostridiales;f_; __; $_{-}$

6.0E-03 P_Firmicutes;c_Clostridia;o_Clostridiales;_;_;_;s

$6.0 \mathrm{E}-03$ P_Firmicutes;c_Clostridia;o_Clostridiales; $\mathrm{f}_{-} ; \mathrm{g}_{-} ; \mathbf{s}_{-}$

6.0E-03 P_Firmicutes;c_Clostridia;o_Clostridiales;_;;__;

2.9E-05 P_Firmicutes;c_Clostridia;o_Clostridiales;_; $; g_{-} ; \mathbf{s}$

4.4E-05 P_Firmicutes;c_Clostridia;o_Clostridiales; $\mathbf{f}_{-} ; \mathbf{g}_{-} ; \mathbf{s}_{-}$

$1.0 \mathrm{E}-06$ p Firmicutes;c Clostridia;o Clostridiales; $;$; ;s

$1.9 E-06$ p_Firmicutes;c_Clostridia;o_Clostridiales; $\mathbf{f}_{-} ; \mathbf{g}_{-} ; \mathbf{s}_{-}$

9.0E-04 P_Firmicutes;C_Clostridia;__Clostridiales;__Christensenellaceae;g_; _

$1.5 \mathrm{E}-03$ P_Firmicutes;c_Clostridia;o_Clostridiales;_CChristensenellaceae;g_;s

2.1E-02 p_Firmicutes;C_Clostridia;o_Clostridiales;__Christensenellaceae; __;

2.3E-11 P_Firmicutes;c_Clostridia;__Clostridiales;__Christensenellaceae;g_;

4.0E-02 P_Firmicutes;C_Clostridia;o_Clostridiales;__Clostridiaceae;g_;

4.1E-02 p_Firmicutes;c_Clostridia;o_Clostridiales;__Clostridiaceae; $g_{-} ; \mathbf{s}$

$2.4 \mathrm{E}-02$ p_Firmicutes;c_Clostridia;o_Clostridiales;_Clostridiaceae; $g_{-} ; \mathbf{s}_{-}$

4.4E-02 P_Firmicutes;c_Clostridia;o_Clostridiales;_LLachnospiraceae;g_;s_

4.0E-04 P_Firmicutes;C_Clostridia;__Clostridiales;__Lachnospiraceae;g_;

3.3E-02 P_Firmicutes;C_Clostridia;o_Clostridiales;__Lachnospiraceae; g_Anaerostipes;s

6.0E-03 P_Firmicutes;C_Clostridia;o_Clostridiales;__Lachnospiraceae;g_Blautia;s_

6.3E-04 P_Firmicutes;c_Clostridia;o_Clostridiales;f_Lachnospiraceae;g_Blautia;s

9.1E-03 P_Firmicutes;C_Clostridia;o_Clostridiales;__Lachnospiraceae;g_Dorea;s

$\begin{array}{lll}9.1 E-03 & \text { P_Firmicutes;c_Clostridia;__Clostridiales;f_Lachnospiraceae; } \\ 1.3 \mathrm{E}-02 & \text { p_Firmicutes;c_Clostridia;o_Clostridiales;f_Ruminococcaceae }\end{array}$

2.8E-02 p_Firmicutes;c_Clostridia;o_Clostridiales;__Ruminococcaceae;g_s

$2.1 \mathrm{E}-02$ P_Firmicutes;c_Clostridia;o_Clostridiales;__Ruminococcaceae;g_;

2.0E-02 P_Firmicutes;c_Clostridia;o_Clostridiales;f_Ruminococcaceae; __;

$2.6 \mathrm{E}-11$ P_Firmicutes;C_Clostridia;o_Clostridiales;__Ruminococcaceae; $\mathbf{g}_{-} ; \mathbf{s}$

2.0 E-02 p_Firmicutes;c_Clostridia;o_Clostridiales;__Ruminococcaceae;g_Oscillospira;s

$2.0 \mathrm{E}-02$ p Firmicutes;c_Clostridia;o_Clostridiales;f Ruminococcaceae;g_Oscillospira;s

$2.8 \mathrm{E}-02$ P_Firmicutes;c_Clostridia;0_Clostridiales;__Ruminococcaceae;g_Ruminococcus;s

3.4E-02 P_Firmicutes;c_Clostridia;_C_Clostridiales;__Ruminococcaceae;g_Ruminococcus;s_

$5.0 E-03$ P_Firmicutes;C_Clostridia;__Clostridiales;_R_Ruminococcaceae;g_Ruminococcus;s_

6.4E-03 P_Firmicutes;C_Clostridia;O_Clostridiales;_Ruminococcaceae;g_Ruminococcus;s_

1.6E-03 P_Firmicutes;___Clostridia;o_Clostridiales;__Ruminococcaceae;g_Ruminococcus;s_

2.3E-03 P_Firmicutes;c_Clostridia;o_Clostridiales;__Ruminococcaceae;g_Ruminococcus;s

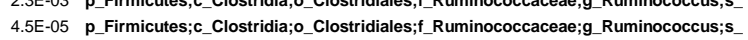

4.4E-02 P_Firmicutes;C_Clostridia;o_Clostridiales;;_Veillonellaceae;g_Veillonella;s_dispar $4.7 \mathrm{E}-02$ P_Proteobacteria;C_Epsilonproteobacteria;o_Campylobacterales;___Helicobacteraceae 


\section{Acetate}

Butyrate

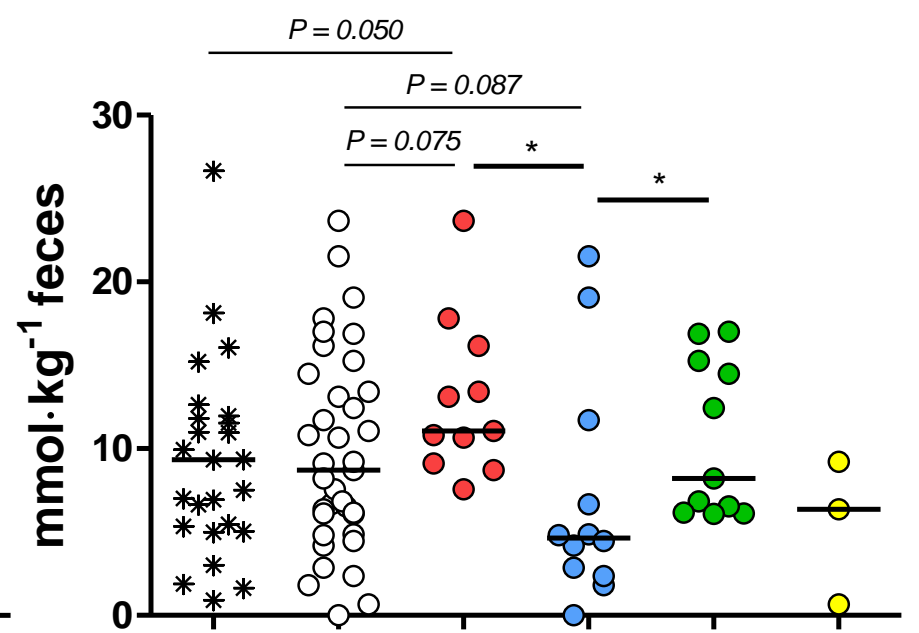

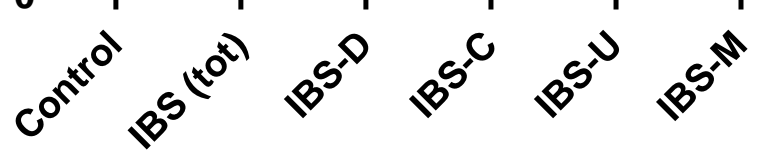

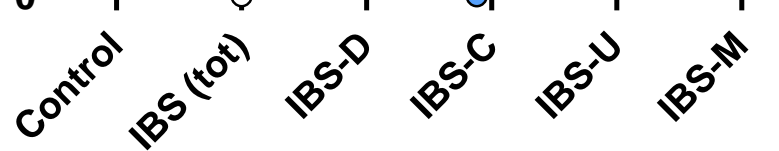

Propionate

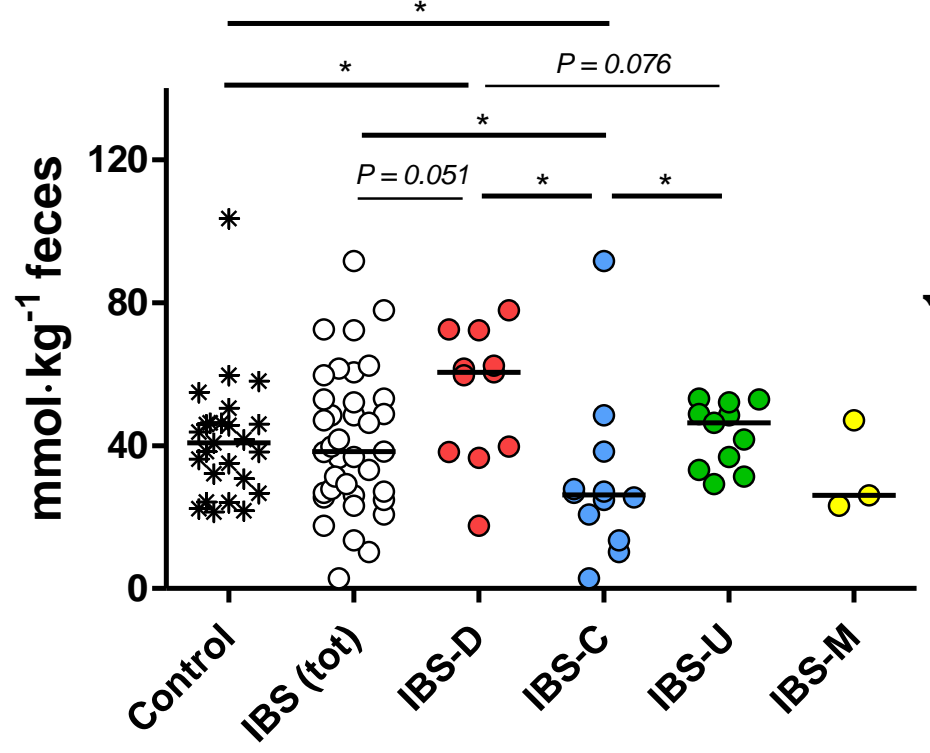

Isobutyrate

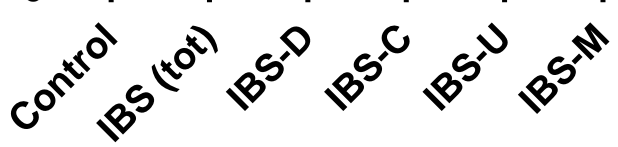

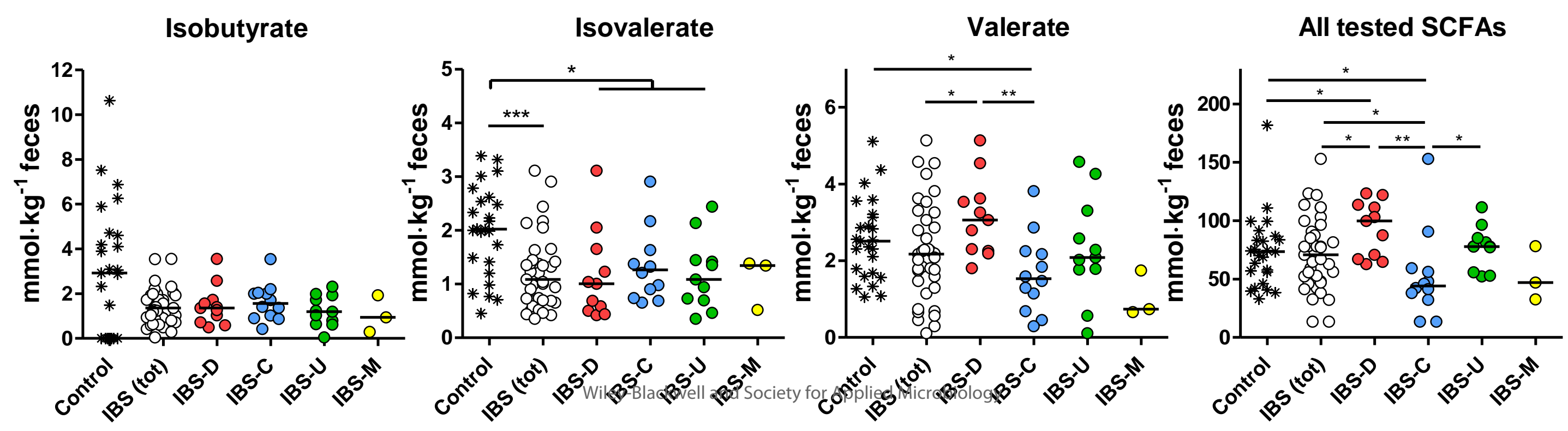

Isovalerate
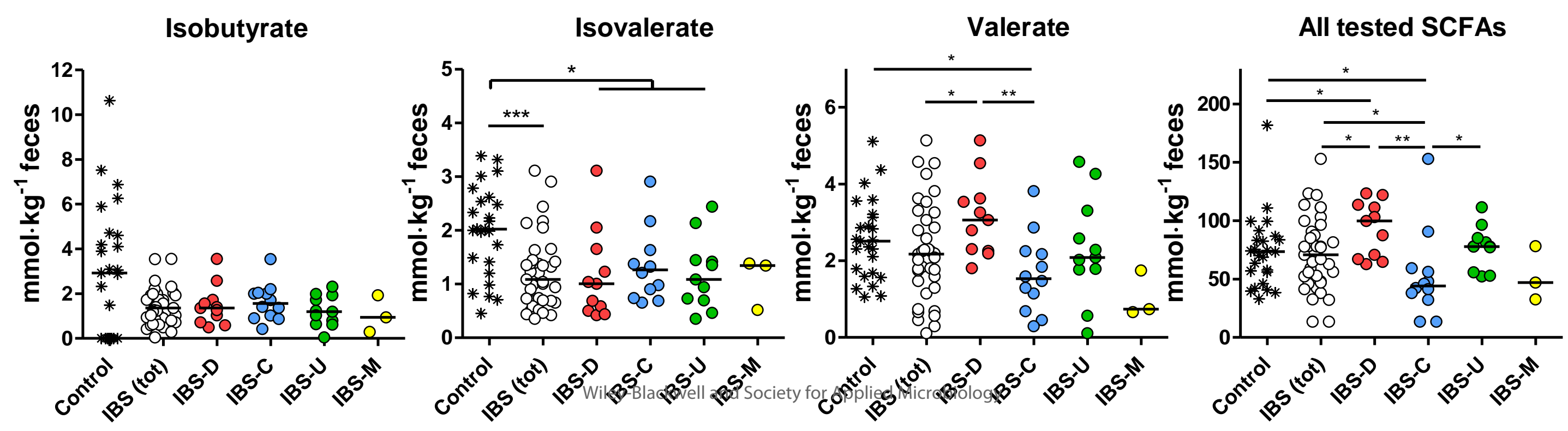

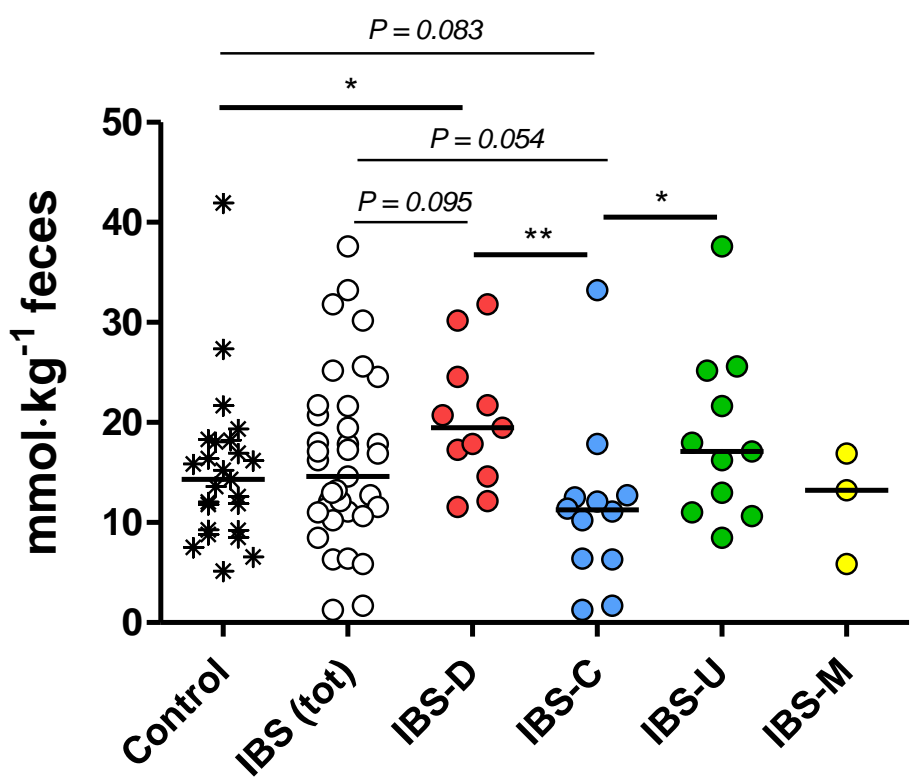









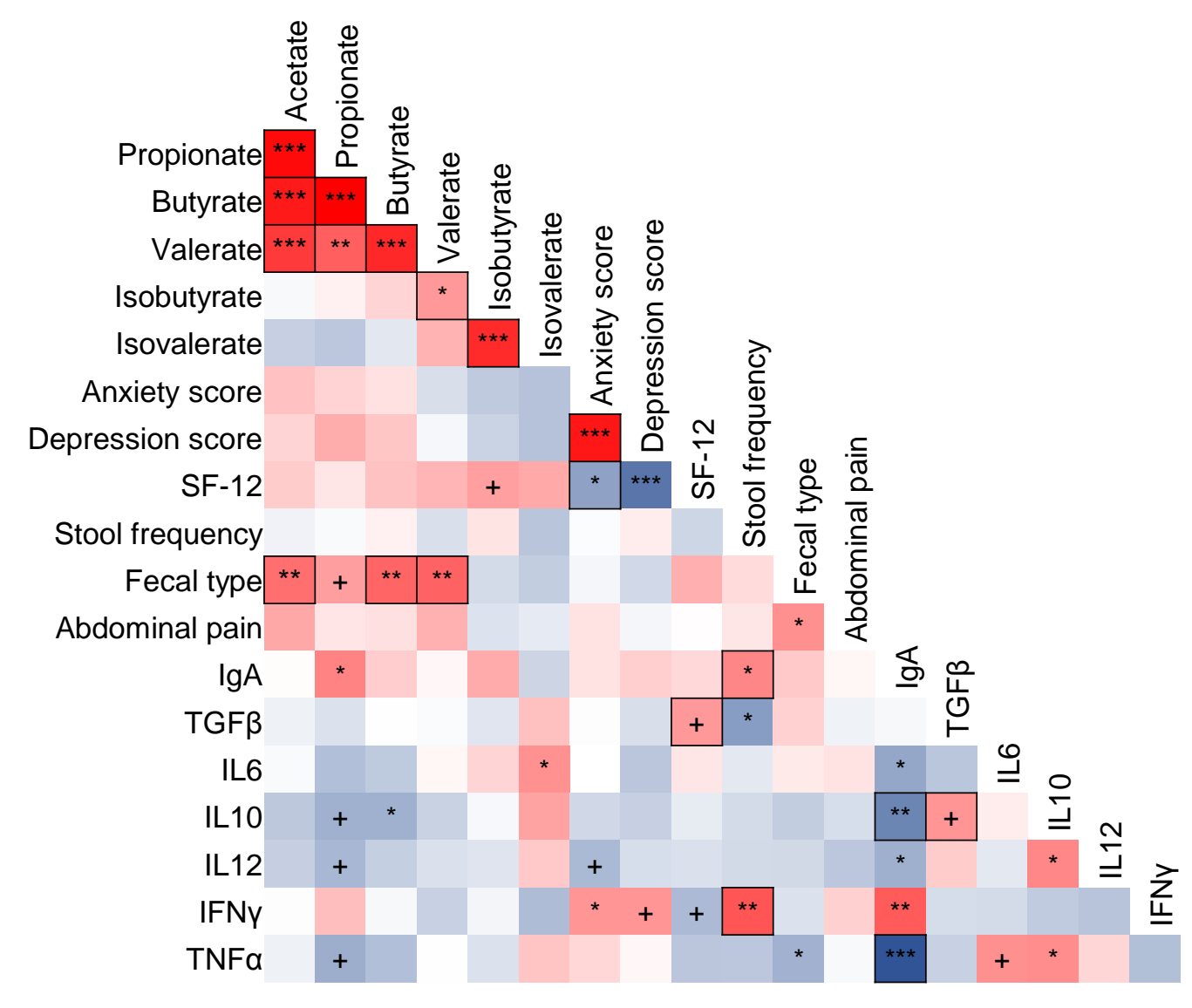

$-0.75$

0.75

Wiley-Blackwell and Society for Applied Microbiology 